\title{
Next-generation hypomethylating agent SGI-110 primes acute myeloid leukemia cells to IAP antagonist by activating extrinsic and intrinsic apoptosis pathways
}

\author{
Jessica Dittmann ${ }^{1} \cdot$ Tinka Haydn $^{1} \cdot$ Patrick Metzger ${ }^{2,3,4} \cdot$ George A. Ward $^{5} \cdot$ Melanie Boerries $^{2,3,6,7} \cdot$ Meike Vogler $^{1}$. \\ Simone Fulda (iD) $1,7,8$
}

Received: 20 May 2019 / Revised: 21 November 2019 / Accepted: 21 November 2019 / Published online: 12 December 2019

(c) The Author(s), under exclusive licence to ADMC Associazione Differenziamento e Morte Cellulare 2019

\begin{abstract}
Therapeutic efficacy of first-generation hypomethylating agents (HMAs) is limited in elderly acute myeloid leukemia (AML) patients. Therefore, combination strategies with targeted therapies are urgently needed. Here, we discover that priming with SGI-110 (guadecitabine), a next-generation HMA, sensitizes AML cells to ASTX660, a novel antagonist of cellular inhibitor of apoptosis protein 1 and 2 (cIAP1/2) and X-linked IAP (XIAP). Importantly, SGI-110 and ASTX660 synergistically induced cell death in a panel of AML cell lines as well as in primary AML samples while largely sparing normal CD34+ human progenitor cells, underlining the translational relevance of this combination. Unbiased transcriptome analysis revealed that SGI-110 alone or in combination with ASTX660 upregulated the expression of key regulators of both extrinsic and intrinsic apoptosis signaling pathways such as TNFRSF1OB (DR5), FAS, and BAX. Individual knockdown of the death receptors TNFR1, DR5, and FAS significantly reduced SGI-110/ASTX660-mediated cell death, whereas blocking antibodies for tumor necrosis factor (TNF)-related apoptosis-inducing ligand (TRAIL) or FAS ligand (FASLG) failed to provide protection. Also, TNFo-blocking antibody Enbrel had little protective effect on SGI-110/ASTX660-induced cell death. Further, SGI-110 and ASTX660 acted in concert to promote cleavage of caspase-8 and BID, thereby providing a link between extrinsic and intrinsic apoptotic pathways. Consistently, sequential treatment with SGI-110 and ASTX660-triggered loss of mitochondrial membrane potential (MMP) and BAX activation which contributes to cell death, as BAX silencing significantly protected from SGI-110/ASTX660-mediated apoptosis. Together, these events culminated in the activation of caspases-3/-7, nuclear fragmentation, and cell death. In conclusion, SGI-110 and ASTX660 cooperatively induced apoptosis in AML cells by engaging extrinsic and intrinsic apoptosis pathways, highlighting the therapeutic potential of this combination for AML.
\end{abstract}

Edited by M. Piacentini Licence

Supplementary information The online version of this article (https:// doi.org/10.1038/s41418-019-0465-8) contains supplementary material, which is available to authorized users.

Simone Fulda

simone.fulda@kgu.de

1 Institute for Experimental Cancer Research in Pediatrics, GoetheUniversity Frankfurt, Frankfurt am Main, Germany

2 Institute of Medical Bioinformatics and Systems Medicine, Medical Center, Faculty of Medicine, University Freiburg, Freiburg im Breisgau, Germany

3 Institute of Molecular Medicine and Cell Research (IMMZ), Albert Ludwigs-University Freiburg, Freiburg im Breisgau, Germany

\section{Introduction}

Acute myeloid leukemia (AML) is a heterogeneous disease characterized by clonal proliferation of poorly differentiated myeloid cells and occurs primarily in older adults

4 Faculty of Biology, University Freiburg, Freiburg im Breisgau, Germany

5 Astex Pharmaceuticals, Cambridge, UK

6 German Cancer Consortium (DKTK), Partner Site Freiburg, Freiburg im Breisgau, Germany

7 German Cancer Research Center (DKFZ), Heidelberg, Germany

8 German Cancer Consortium (DKTK), Partner Site Frankfurt, Frankfurt am Main, Germany 
(age $\geq 60$ years) [1]. Despite improvements in outcomes for younger AML patients in recent decades, the prognosis for older patients, who are ineligible for intensive treatment, remains dismal [2], highlighting the urgent need for better therapeutic options.

Dysfunction of epigenetic modifiers, such as DNA methyltransferases (DNMTs), contributes to AML pathogenesis through aberrant epigenetic silencing of tumor suppressor genes (TSGs) involved in differentiation and apoptosis [3]. DNMT3A mutations are found in $22 \%$ of AML patients, and around $60 \%$ of these mutations affect the R882 codon, which is highly associated with poor prognosis [3, 4]. Firstgeneration hypomethylating agents (HMAs), such as azacytidine and decitabine, have been approved for the treatment of older AML patients [5]. SGI-110 (guadecitabine), a dinucleotide of decitabine and deoxyguanosine, is a nextgeneration HMA that is resistant to degradation by cytidine deaminase and provides a prolonged in vivo exposure compared with decitabine [6]. Recently, safety and clinical activity of SGI-110 in both elderly treatment-naive and relapsed/refractory AML patients have been shown in phase II trials $[7,8]$. The proposed central mechanism of action of HMAs is the depletion of DNMTs, thus inducing hypomethylation of global DNA and CpG-island promoters, which might lead to gene expression of silenced TSGs [6,9], and may sensitize tumor cells to other anticancer agents, including chemotherapeutics [10, 11], immunotherapeutics [12], or apoptosis-inducing agents [13].

Apoptosis plays an important role in the hematopoietic system. There are two well-defined pathways of apoptosis: the extrinsic and the intrinsic pathway [14]. The extrinsic (death receptor-mediated) pathway is activated upon interaction of death receptor ligands, such as tumor necrosis factor (TNF), TNF-related apoptosis-inducing ligand (TRAIL), and FAS ligand (FASLG) with their cognate death receptors TNF receptor 1 (TNFR1), TRAIL-R1 (DR4), TRAIL-R2 (DR5), and FAS, resulting in the activation of caspase- 8 , which can cleave downstream effector caspases [15]. The intrinsic (mitochondrial-mediated) pathway involves loss of mitochondrial membrane potential (MMP) due to mitochondrial outer membrane permeabilization (MOMP) that is controlled by proapoptotic (e.g., BAX, BAK, and BID) and antiapoptotic proteins of the BCL-2 family. This leads to caspase activation, nuclear fragmentation, and apoptotic cell death [14]. A crosslink between extrinsic and intrinsic pathways is provided by caspase-8-mediated cleavage of BID into its active form (tBID) [16].

Evasion of apoptosis is a major cause of treatment resistance and is often caused by overexpression of antiapoptotic proteins such as inhibitor of apoptosis proteins (IAPs) [17]. Overexpression of XIAP has been associated with poor outcome in AML [18] and high cIAP1 expression has been reported in pediatric AML [19]. XIAP exerts its antiapoptotic activity by inhibiting caspases [20], while cIAP1/2 can regulate proapoptotic signaling complexes [17]. Therefore, IAPs are considered attractive targets for anticancer therapy. Various IAP antagonists have been developed which trigger autoubiquitination and proteasomal degradation of IAPs [21]. ASTX660, a novel orally bioavailable, nonpeptidomimetic antagonist of cIAP1/2 and XIAP [22], is currently under evaluation in a phase I/II clinical trial for advanced solid tumors and lymphomas (NCT: 02503423). However, several IAP antagonists have so far shown only weak single-agent efficacy in clinical trials [22].

Since the therapeutic efficacy of HMAs is limited in AML patients [13], there is a high medical need to identify novel combinations. Therefore, in the present study we investigated whether priming with next-generation HMA SGI-110 increases the sensitivity of AML cells towards cell death triggered by the novel IAP antagonist ASTX660.

\section{Methods and materials}

\section{AML cell lines}

AML cell lines were obtained from German Collection of Microorganisms and Cell cultures (DSMZ, Braunschweig, Germany), except ML-2 and PLB-985 cells that were kindly provided by T. Oellerich, Department of Medicine II, University Hospital Frankfurt, Germany. All cell lines were authenticated by STR profiling and routinely checked for mycoplasma contamination. All AML cell lines except OCI-AML-3 were cultured in RPMI 1640 medium (Life Technologies, Inc., Darmstadt, Germany) supplemented with $10 \%$ fetal calf serum (FCS), $1 \%$ penicillin/streptomycin, and $1 \%$ sodium pyruvate (Invitrogen, Karlsruhe, Germany). OCI-AML3 cells were cultured in alpha-MEM medium supplemented with $20 \%$ FCS, $1 \%$ penicillin/streptomycin, and $1 \%$ sodium pyruvate.

\section{Primary samples}

Bone marrow specimens from AML patients were obtained at diagnosis before the onset of therapy after obtaining written informed consent from patients according to the declaration of Helsinki and after approval by the local ethics committee of the University Hospital Frankfurt (Approval No. SHO-05-2014). Mononuclear cell (MNC) fractions were obtained by density gradient centrifugation using Ficoll Isopaque (Amersham Bioscience, Freiburg, Germany) and maintained as previously described [23]. The clinical characteristics of the AML patients are summarized in Supplementary Table S1. Primary samples with spontaneous cell death $\geq 40 \%$ at the time point of measurement were excluded from the analysis. MNCs and clinical data 
were obtained from the hematological biobank and the tumor documentation of the UCT Frankfurt, Germany.

Human G-CSF-mobilized CD34+ hematopoietic progenitor cells (HPCs) from healthy donors were kindly provided by $\mathrm{H}$. Bönig, Institute for Transfusion Medicine and Immunohematology, Frankfurt, Germany. After thawing, HPCs were cultured in IMDM enriched with $20 \%$ FCS, $1 \%$ penicillin/streptomycin, $50 \mathrm{ng} / \mathrm{ml} \mathrm{rh}-\mathrm{SCF}, 50 \mathrm{ng} / \mathrm{ml}$ IL-3, $100 \mathrm{ng} / \mathrm{ml}$ FLT3-Ligand (AF-300-19, PeproTech), and $20 \mathrm{ng} / \mathrm{ml} \mathrm{GM-CSF}$.

\section{Determination of cell death and apoptosis}

For measurements of cell death and apoptosis, cells were cultured at $1 \times 10^{5}$ cells $/ \mathrm{ml}$ (AML cell lines) or $1 \times 10^{6}$ cells $/ \mathrm{ml}$ (primary AML, CD34+ HPCs). Cell death of suspension cells was assessed by forward/side scatter (FSC/SSC) and flow cytometry (FACS Canto II, BD Biosciences, Heidelberg, Germany). Apoptosis was determined by FACS analysis following staining of the cells with Annexin V-FITC and propidium iodide (PI). Early-apoptotic (Annexin $\mathrm{V}+\mathrm{PI}-$ ) and late-apoptotic cells (Annexin $\mathrm{V}+/ \mathrm{PI}+$ ) were summarized as Annexin V+ cells. Primary AML cells were stained with Annexin V-FITC and $0.5 \mu \mathrm{l}$ anti-CD45-APC antibody (17-0459-42, eBioscience, San Diego, CA, USA). Flow cytometry was used to determine cell death of primary AML blasts identified by using a CD45/SSC gating strategy as described previously [24]. Flow cytometric analysis using a PI-containing Nicoletti buffer was performed to determine the proportion of apoptotic sub-G1 hypodiploid cells with internucleosomal DNA fragmentation as previously described [25].

\section{DNA isolation and global methylation assay}

DNA was isolated using the PureLink Genomic DNA Mini Kit (Invitrogen, Carlsbad, CA, USA). Global DNA Methylation-long interspersed nuclear element-1 (LINE-1) kit (Active Motif, Carlsbad, CA, USA) was employed in accordance with the manufacturer's instructions.

\section{Human genome microarray profiling}

RNA was isolated using the PeqGold Total RNA Kit and the PeqGOLD DNAase digest kit (Peqlab, Erlangen, Germany) following the manufacturer's protocol. Human genome microarray profiling was performed at the DKFZ Genomics and Proteomics Core Facility (Heidelberg, Germany) using the Affymetrix Human Genome U133 Plus 2.0 Array.

\section{Transcriptome analysis}

Raw Affymetrix cell files were analyzed using the $\mathrm{R} /$ Bioconductor [26, 27] package affy [28] followed by quantile, robust multichip analysis (RMA) background normalization and $\log 2$ transformation. After preprocessing and filtering, 20352 genes were further analyzed. Differentially expressed genes (DEGs) were identified with the R/Bioconductor package limma [29]. For the comparisons of SGI-110/ASTX660-Ctrl, SGI-110-Ctrl, SGI-110ASTX660 and SGI-110/ASTX660-ASTX660 the leastsquares method and for the comparisons of ASTX-Ctrl and SGI-110/ASTX660-SGI-110 the robust method for linear model fitting were used. Genes were considered significant with an adjusted $p$ value $<0.05$ (Benjamini-Hochberg). Microarray data were deposited in the publicly accessible database Gene Expression Omnibus under accession number GSE138322.

\section{Gene-set enrichment analysis (GSEA)}

Enrichment of signaling pathways was performed as implemented in the R/Bioconductor package [30] with Gene ontology (GO) terms [31, 32], Reactome pathways [33] and ConsensusPathDB pathways [34]. Pathways are considered significant with an adjusted $p$ value $<0.05$ (Benjamini-Hochberg).

\section{Cell surface expression of death receptors}

Flow cytometric analysis of cell surface expression of TNFR1, DR4, DR5, and FAS was performed using following phycoerythrin (PE)-conjugated antibodies: anti-TNFR1 (130-106-286, Miltenyi Biotech, Bergisch Gladbach, Germany), anti-DR4 (FAB347P), anti-DR5 (FAB6311P, R \& D Systems, Wiesbaden, Germany) and anti-FAS (556641, BD BioSciences, San Diego, CA, USA) and their respective isotype controls (IgG1 (IC002P), IgG2b (IC0041P, R \& D systems, Wiesbaden, Germany)), REA control (130-113-462, Miltenyi Biotech, Bergisch Gladbach, Germany). In brief, treated cells were harvested and washed twice with ice-cold staining buffer (2\% FCS in PBS). Afterwards, cells were incubated with PE-conjugated antibodies to the death receptors or respective isotype controls for $30 \mathrm{~min}$ at $4{ }^{\circ} \mathrm{C}$ in the dark. Cells were then washed twice with staining buffer and resuspended in PBS for flow cytometric analysis. Data were analyzed using FlowJo software.

\section{Immunoprecipitation}

BAX activation was determined by immunoprecipitation using active conformation-specific antibodies. Briefly, cells were lysed in CHAPS buffer (1\% CHAPS, $150 \mathrm{mM}$ $\mathrm{NaCl}, 10$ mM HEPES, pH 7.4) supplemented with protease inhibitor cocktail (1169749800, Roche, Mannheim, Germany). Briefly, $500 \mu \mathrm{g}$ protein was immunoprecipitated with $2 \mu \mathrm{l}$ mouse anti-BAX antibody (6A7, Sigma-Aldrich, 
Munich, Germany) and $10 \mu$ l pan-mouse IgG Dynabeads (Dako, Hamburg, Germany) overnight at $4{ }^{\circ} \mathrm{C}$ and washed with CHAPS buffer. The precipitate was analyzed for BAX expression by Western blotting, using the rabbit antiBAX NT antibody (ABC11, Merck-Millipore, Burlington, MA, USA).

XIAP immunoprecipitation was performed as previously described [22] with the following exception: cell lysates were incubated with streptavidin-coated Dynabeads (60210, Thermo Fisher Scientific). The resulting precipitate was analyzed by Western blotting and the membranes probed with antibodies against second mitochondrial activator of caspases (SMAC) (\#2954) and XIAP (\#14334, Cell Signaling Technology) to detect interactions.

\section{Determination of MMP}

To measure MMP cells were incubated with $100 \mathrm{ng} / \mathrm{ml}$ tetramethylrhodamine methyl ester (TMRM Reagent; Thermo Fisher Scientific, Waltham, MA, USA) for $10 \mathrm{~min}$ at $37^{\circ} \mathrm{C}$, washed and directly analyzed by flow cytometry.

\section{Western blot analysis}

Cells were lysed using a Triton X-100 lysis buffer ( $30 \mathrm{mM}$ TrisHCl, $150 \mathrm{mM} \mathrm{NaCl}, 10 \%$ Glycerol, $0.5 \mathrm{mM}$ PMSF, 2 mM DTT, $1 \%$ Triton X-100, and $1 \times$ Protease Inhibitor Cocktail). Western blot analysis was carried out using the following primary antibodies: rabbit anti-BID (2002S, Cell Signaling), rabbit anti-caspase-8 (ab32125, Abcam), goat anti-cIAP1 (\#AF8181, R \& D systems), rabbit anti-BAX (ABC11, Merck-Millipore), rabbit anti-BAK (06-536, Upstate/Merck), mouse anti-XIAP (610716, BD Bioscience), rabbit anti-DNMT1 (39905, Active motif), mouse anti-DNMT3A (sc-365769, Santa Cruz Biotechnology), mouse anti-GAPDH (5G4-6C5, BioTrend (Hy Test Ltd)), mouse anti- $\beta$-ACTIN (A5441, Sigma), and rat anticIAP2 (ALX-803-341-C100, Enzo Life Sciences, Farmingdale, NY, USA). Goat anti-mouse IgG, goat anti-rabbit IgG (Abcam), goat anti-rat IgG, donkey anti-goat IgG conjugated to horseradish peroxidase (Santa Cruz Biotechnology, Santa Cruz, CA, USA), and enhanced chemiluminescence (Amersham Bioscience, Freiburg, Germany) or infrared dye-labeled secondary antibodies and infrared imaging (Odyssey Imaging System, LI-COR Bioscience, Bad Homburg, Germany) were used for detection. Representative blots of at least two independent experiments are shown.

\section{RNA interference}

Gene silencing with small interfering RNA (siRNA) was achieved using Silencer Select siRNA (Thermo Fisher
Scientific, Waltham, MA, USA) and Neon Transfection System (Invitrogen, Karlsruhe, Germany) following the manufacturer's protocol. The following constructs were used: nontargeting control siRNA (4390843) or targeting siRNAs for BAX (\#1: s1888, \#2: s1890), BAK (\#1: s1880, \#2: s1881), cIAP1 (\#1: s1449, \#2: s1450, \#3: s1448), XIAP (\#1: s1454, 2: s1555, \#3: s1556), TNFR1 (\#1: s14266, \#2: s14267), DR5 (\#1: s16756, \#2: s16758), and FAS (\#1: s1506, \#2: s1507, \#3: s1508). For knockdown of cIAP1 and XIAP, cells were transfected once with $200 \mathrm{nM}$ siRNA and immediately treated with SGI110. For all other knockdowns, cells were transfected twice with $100 \mathrm{nM}$ siRNA and treated $24 \mathrm{~h}$ after the second transfection.

\section{Analysis of caspase-3/-7 activity and of morphological changes of the nucleus}

Caspase activity was determined using Cell Event Caspase-3/-7 Green Detection Reagent (Thermo Fisher Scientific) according to manufacturer's instructions. For analysis by ImageXpress Micro XLS system (Molecular Devices, Biberach an der Riss, Germany), cells were additionally counterstained with Hoechst-33342 (Invitrogen, Carlsbad, CA, USA). For evaluation of the nuclear morphology, cells were stained with Hoechst33342 and examined using an inverted fluorescence microscope (ImageXpress Micro XLS system) followed by analysis with MetaXpress Software (Molecular Devices Sunnyvale, CA, USA) using the nuclear fragmentation scoring tool.

\section{Statistical analysis}

All results are expressed as mean and standard deviation (SD). The numbers of independent repetitions and replicates for each experiment are indicated in the respective figure legends. Experiments were considered as reliable if the SD did not exceed 10\% within the replicates and repetitions. For each in vitro independent experiment using cancer cell lines, technical triplicates were used and three experiments were performed to ensure adequate statistical power. Statistical significance was verified by using $t$-test in Microsoft Excel (two-samples, two-tailed distribution, unequal variance), unless otherwise indicated. For drug combination dose-response studies in multiple AML cell lines, data were analyzed by one-way ANOVA followed by Tukey's multiple comparisons test, using GraphPad Prism as specified in the table legend. Drug interaction was analyzed using CalcuSyn software (Biosoft, Cambridge, UK) [35]. The obtained values were rated as follows: $\mathrm{CI}<0.9$ indicates synergism, 0.9-1.1 additivity and $>1.1$ antagonism. 
Fig. 1 SGI-110 synergizes with ASTX660 to induce cell death and to suppress clonogenic growth of AML cell lines. a Following pretreatment with indicated concentrations of SGI-110 for $24 \mathrm{~h}$, AML cells were treated with indicated concentrations of ASTX660 for another $48 \mathrm{~h}$. Cell death was determined by FSC/ SSC analysis and flow cytometry. b After $24 \mathrm{~h}$ of pretreatment with SGI-110 (MOLM-13: $50 \mathrm{nM}, \mathrm{ML}-2$ : $2 \mu \mathrm{M})$, AML cells were treated with ASTX660 (MOLM-13: 40 $\mu \mathrm{M}, \mathrm{ML}-2: 5 \mu \mathrm{M})$ for indicated time points. Cell death was determined by FSC/SSC analysis and flow cytometry. Significances after combination treatment are calculated versus single-treated cells. c AML cells were exposed to SGI-110 (MOLM-13: $50 \mathrm{nM}$, ML-2: $0.5 \mu \mathrm{M}$ ) for $24 \mathrm{~h}$ followed by ASTX660 (MOLM-13: $40 \mu \mathrm{M}$, ML-2: $5 \mu \mathrm{M}$ ) for $15 \mathrm{~h}$ before incubation in methylcellulose. Colony formation was assessed after 10-13 days and the number of colonies is expressed as percentage of solvent-treated controls. Mean and SD of three independent experiments performed in triplicate are shown. $* p<0.05, * * p<0.01$; $* * * p<0.001$.
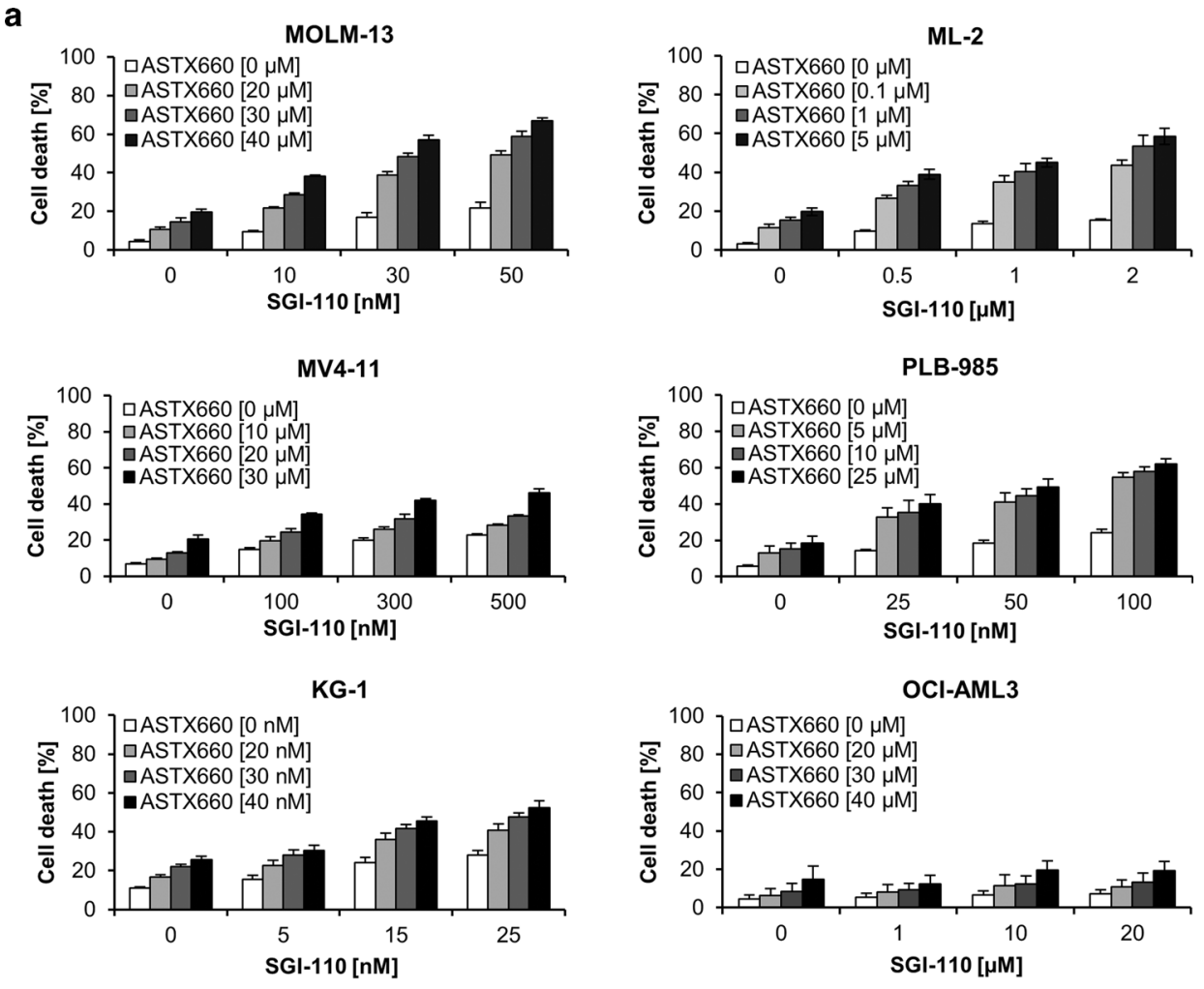

b

MOLM-13

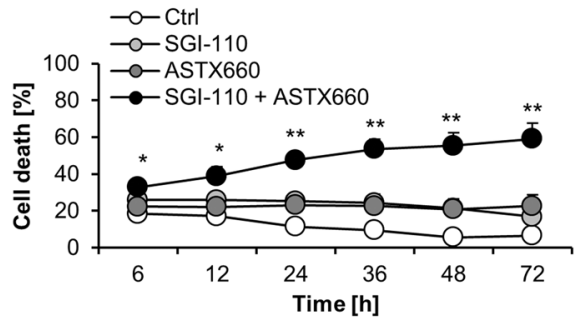

ML-2

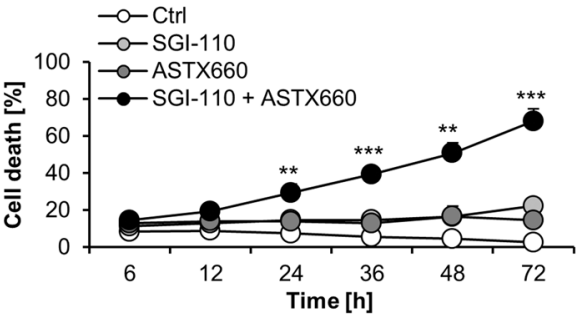

C

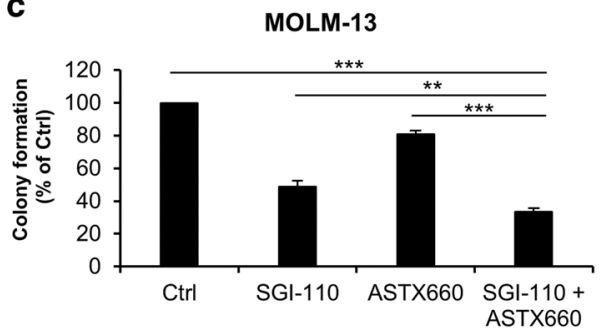

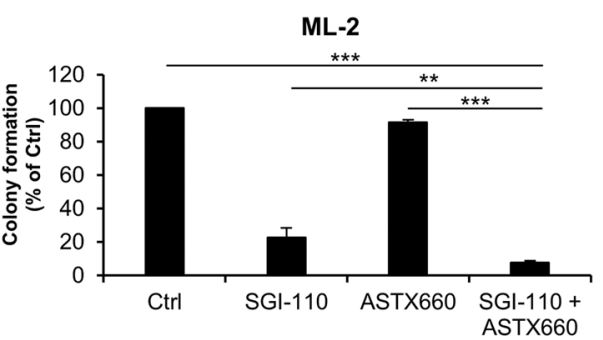

\section{Results}

\section{SGI-110 synergizes with ASTX660 to induce cell death and to suppress clonogenic growth of AML cell lines}

Searching for new clinically relevant drug combinations for the treatment of AML, we asked the question whether the next-generation HMA SGI-110 can prime AML cells towards cell death induced by the novel IAP antagonist ASTX660. Interestingly, dose-response experiments revealed that SGI110 pretreatment synergistically interacted with ASTX660 to induce cell death in five out of six AML cell lines with various genetic backgrounds and representing different AML subtypes (Fig. 1a, Supplementary Table 2-3). Only OCI-AML3 cells that harbor the hotspot $D N M T 3 A^{\mathrm{R} 882 \mathrm{C}}$ mutation did not 
a

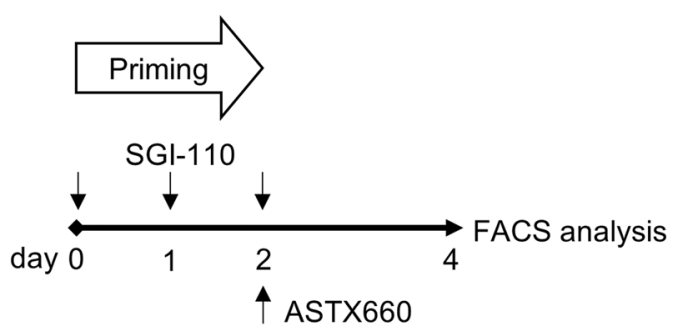

AML \#2

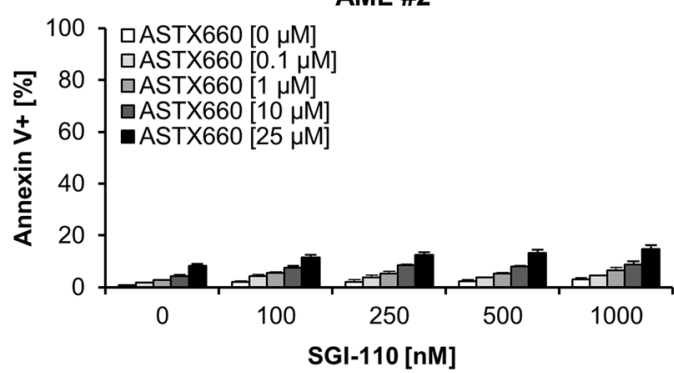

AML \#4

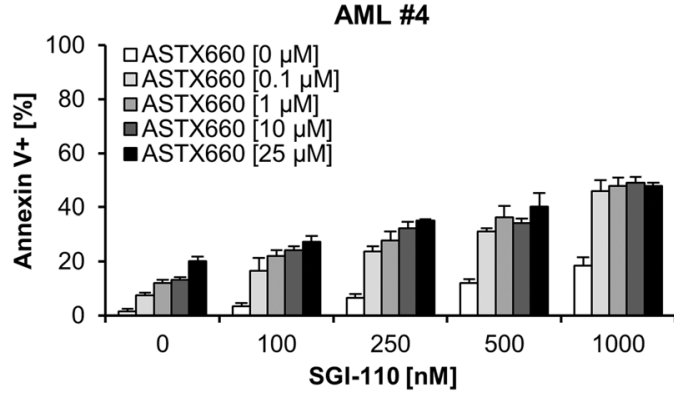

C

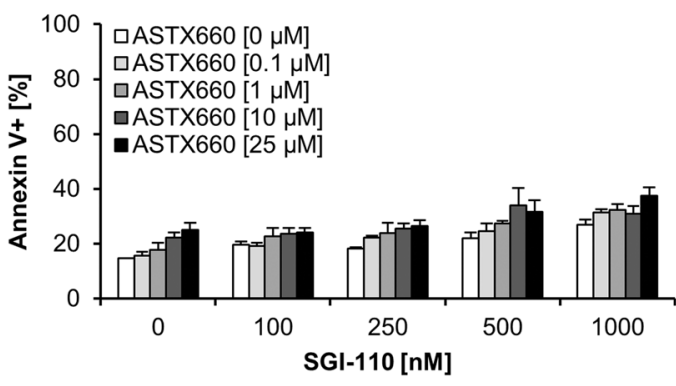

CD34+ HPCs \#3

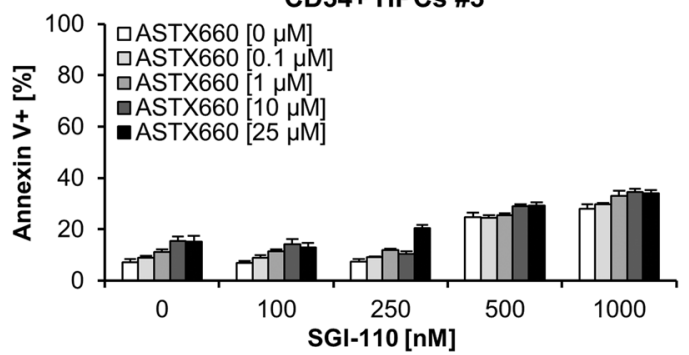

respond even to high concentrations of ASTX660 and SGI110. Synergistic drug interaction was evidenced by calculation of combination index (CI) (Supplementary Table S4). b

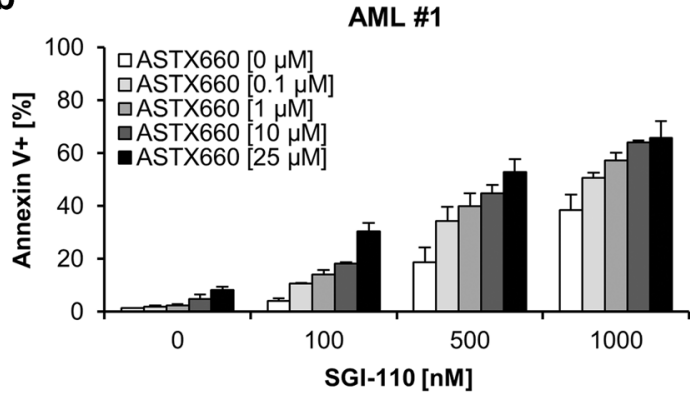

AML \#3

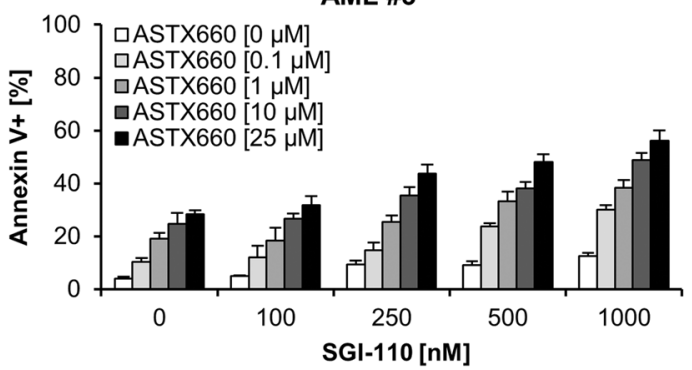

AML \#5

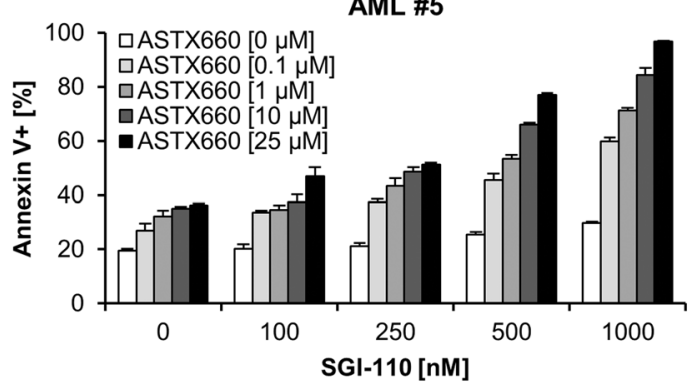

CD34+ HPCs \#2

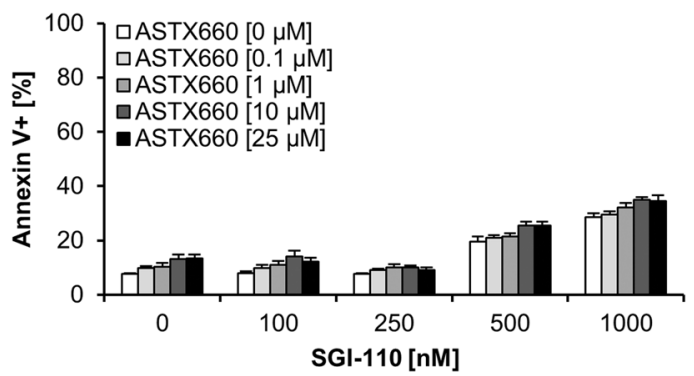

MOLM-13 and ML-2 cells, representing the most common AML subtypes, were selected for further experiments. To investigate whether the timing of drug treatment schedules 
Fig. 2 SGI-110 cooperates with ASTX660 to induce cell death in a large proportion of primary $A M L$ blasts while showing minimal toxicity against normal CD34+ HPCs. a Treatment schedule for primary AML blast cells and CD34+ HPCs. Primary cells were primed with different concentrations of SGI-110 $(0.1-1 \mu \mathrm{M})$ three times at $24 \mathrm{~h}$ intervals, followed by different concentrations of ASTX660 $(0.1-25 \mu \mathrm{M})$ on day 2. Cell death was determined by flow cytometric analysis on day 4 . Stimulation of the primary AML samples (b) and CD34+ HPCs (c) with SGI-110 and/or ASTX660 was performed according to our treatment schedule (a). b Combined Annexin V-FITC/CD45-APC staining and flow cytometry were used to determine cell death of primary AML blasts identified by CD45/SSC gating procedure. c Apoptotic cell death in normal CD34+ HPCs was determined by Annexin V-FITC/PI staining and flow cytometry. b, c Mean and SD of single experiments performed in triplicate are shown.

influences drug interactions, drug combinations were hence further tested using simultaneous application. This treatment schedule induced up to $20 \%$ less cell death in MOLM-13 and ML-2 cells than the sequential treatment with SGI-110 and ASTX660 (Supplementary Fig. S1, Supplementary Table S3, S5), demonstrating that priming by SGI-110 increases the efficacy of ASTX660 treatment.

Time-dependent analysis of drug-induced cell death demonstrated that sequential treatment with SGI-110 and ASTX660 increased cell death over time (Fig. 1b). Besides these short-term assays, we tested the drug combination on long-term clonogenic survival by performing methylcellulose colony forming assays. Importantly, SGI-110 and ASTX660 cooperated to significantly reduce colony formation compared with single-agent treatments (Fig. 1c; Supplementary Fig. S2). In order to further investigate the effect of sequential SGI110/ASTX660 treatment on cell proliferation, we analyzed cell growth by viable cell counting as well as immunofluorescence staining of the proliferation marker Ki-67 [36]. In line with the results of the colony formation assay, SGI-110 alone, or even more pronounced in combination with ASTX660, dramatically reduced proliferation of ML-2 cells, whereas MOLM-13 cells were only slightly affected (Supplementary Fig. S3A-B). Taken together, SGI-110 synergistically acted in concert with ASTX660 to induce cell death and to suppress proliferation and long-term clonogenic growth.

\section{SGI-110/ASTX660 regimen is synergistically active against primary AML blasts, whereas it shows minimal toxicity against CD34 + HPCs}

To assess the translational relevance of our findings we extended our studies to freshly isolated leukemic blasts from treatment-naive AML patients, which were pretreated with SGI-110 for 3 consecutive days, whereby ASTX660 was added on day 2 (Fig. 2a). SGI-110 concentrations were chosen according to plasma levels reported in patients [37]. Importantly, combined exposure to SGI-110 and ASTX660 substantially increased cell death in four out of five AML samples, whereas single-agent treatment with either SGI-110 or ASTX660 had minimal to moderate effects on cell death (Fig. 2b). Calculation of CI values revealed that sequential treatment with SGI-110 and ASTX660 synergistically induced cell death in most primary AML samples (Supplementary Table S6).

In contrast to primary AML blasts, the identical treatment failed to synergize in the induction of cell death in normal CD34+ HPCs from three healthy donors (Fig. 2c), whereas standard and high doses of Cytarabine, based on therapeutically achievable plasma concentrations during standard and high-dose therapy $[38,39]$, were highly toxic to normal CD34+ HPCs (Supplementary Fig. S4). Also, the sequential SGI-110/ASTX660 treatment did not affect long-term clonogenic survival of CD34+ HPC (Supplementary Fig. S5A), while Cytarabine inhibited colony formation in a dose-dependent manner (Supplementary Fig. S5B). Taken together, these data suggest that sequential SGI-110/ASTX660 treatment exerts some tumor selectivity against AML blasts with minimal effects on normal CD34+ HPCs pointing to a potential therapeutic window.

\section{SGI-110 and ASTX660 demonstrate on-target activity in AML cell lines}

Next, we monitored on-target activity of SGI-110 and ASTX660. DNMT1 and DNMT3A protein levels were depleted within $24 \mathrm{~h}$ of SGI-110 pretreatment and did not recover until $48 \mathrm{~h}$ of sequential treatment with ASTX660, confirming that SGI-110 directly targets DNMTs for protein degradation (Fig. 3a). As depletion of DNMT activity results in global hypomethylation of the genome, we further investigated changes in global DNA methylation. Indeed, SGI-110 significantly caused hypomethylation of LINE-1, which serves as a surrogate marker for global DNA methylation [40], within $24 \mathrm{~h}$ of SGI-110 pretreatment (Fig. 3b). As IAP antagonists have been reported to stimulate autoubiquitination and proteasomal degradation of IAP proteins [21], we examined the effect of ASTX660 on IAP protein levels. As both model cell lines do not express cIAP2 (Supplementary Fig. S6), we focused on the antagonism of cIAP1 and XIAP by ASTX660 treatment. ASTX660 induced degradation of cIAP1 in the absence and presence of SGI-110, while XIAP protein levels remained relatively unaffected, as expected (Fig. 3c) [22]. To further confirm the direct antagonism of XIAP by ASTX660 we also assessed the displacement of SMAC from XIAP by 
a

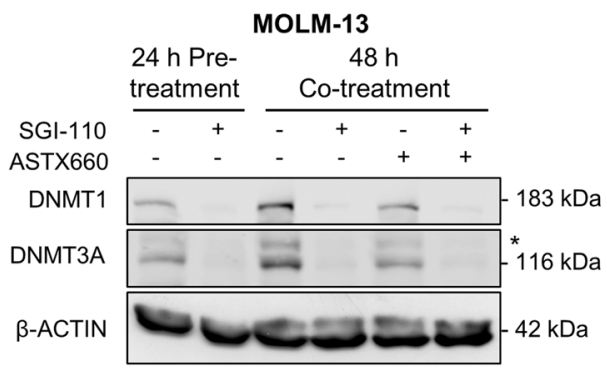

b

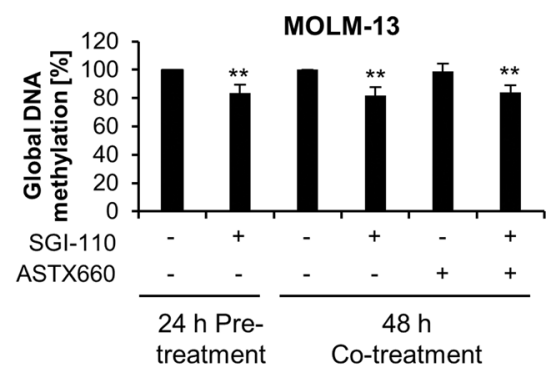

C

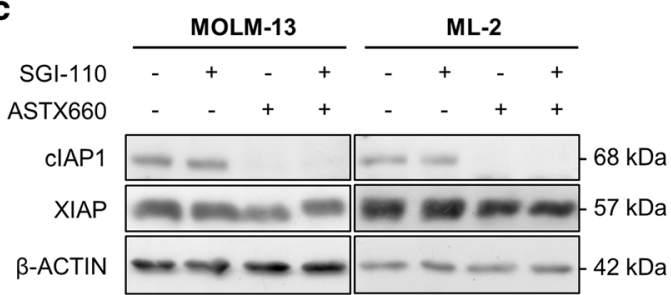

ML-2

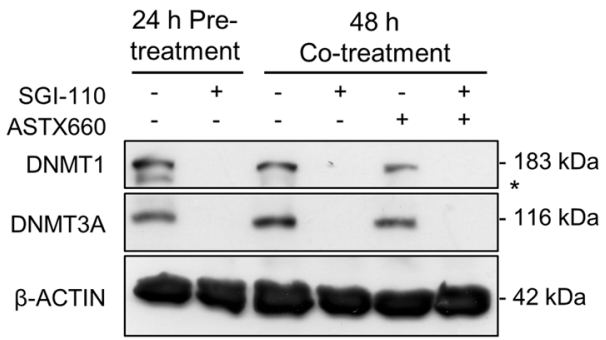

ML-2

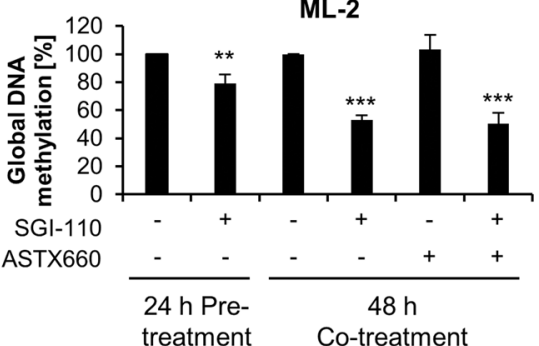

d

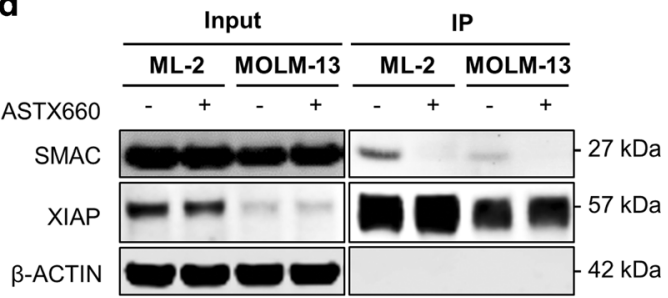

e

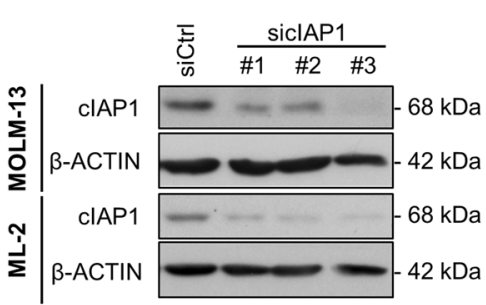

g

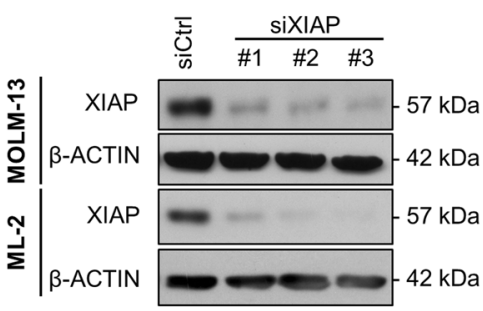

f
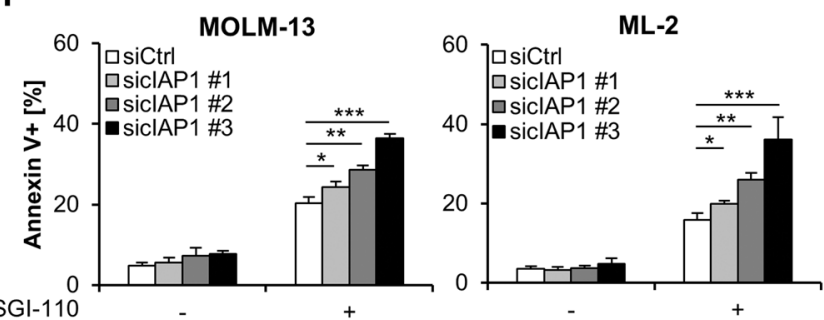

h

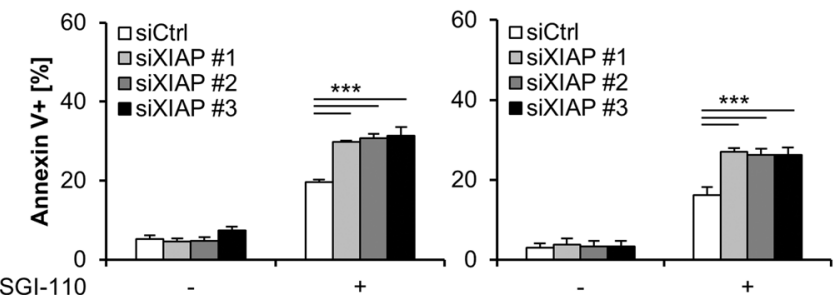

immunoprecipitation using anti-XIAP antibody. Following ASTX660 treatment for $4 \mathrm{~h}$, a complete disappearance of SMAC previously immunoprecipitated with XIAP was observed in MOLM-13 and ML-2 cells (Fig. 3d). These results confirm on-target activity of SGI-110 as well as of ASTX660 in AML cell lines.
Since ASTX660 can antagonize both cIAP1 and XIAP [22], we individually silenced cIAP1 and XIAP to define their contribution to the observed synergistic interaction with SGI-110. The efficiency of cIAP1 and XIAP knockdown by several independent siRNAs was confirmed by Western blotting (Fig. 3e, g). Of note, silencing of cIAP1 
Fig. 3 SGI-110 and ASTX660 demonstrate on-target activity in AML cell lines. a, b Following $24 \mathrm{~h}$ of pretreatment with SGI-110 (MOLM-13: $50 \mathrm{nM}$, ML-2: $2 \mu \mathrm{M}$ ), AML cells were treated with ASTX660 (MOLM-13: $40 \mu \mathrm{M}$, ML-2: $5 \mu \mathrm{M}$ ) for $48 \mathrm{~h}$. a Protein expression of DNMT1 and DNMT3A was determined at indicated time points by Western blotting. $\beta$-ACTIN was used as loading control. Asterisks (*) mark unspecific bands. b Global DNA methylation was determined by colorimetric quantification of methylation levels of LINE-1, a widely used surrogate marker of global DNA methylation, and is shown as percentage of solvent-treated control cells. c Following $24 \mathrm{~h}$ of pretreatment with SGI-110 (MOLM-13: $50 \mathrm{nM}, \mathrm{ML}-2$ : $2 \mu \mathrm{M}$ ), AML cells were treated with ASTX660 (MOLM-13: $40 \mu \mathrm{M}$, ML-2: $5 \mu \mathrm{M}$ ) for $24 \mathrm{~h}$. Protein levels of cIAP1 and XIAP were assessed by Western blotting. $\beta$-ACTIN served as loading control. d AML cells were treated for $4 \mathrm{~h}$ with ASTX660 (MOLM-13: $40 \mu \mathrm{M}$, ML-2: $5 \mu \mathrm{M})$. Cell lysates were subjected to immunoprecipitation using anti-XIAP antibody. Input lysates and precipitates were analyzed for XIAP and SMAC expression by Western blotting. $\beta$-Actin served as loading control. AML cells were transfected with siRNA against cIAP1 (e, f), XIAP (g, h) or non-targeting siRNA (siCtrl). Expression levels of cIAP1 (e) and XIAP (g) were assessed by Western blotting, with $\beta$-ACTIN serving as loading control. $\mathbf{f}, \mathbf{h}$ To analyze the effect of siRNA-mediated loss of cIAP1 and XIAP on cell death, transfected cells were immediately treated with SGI-110 (MOLM-13: 50 nM, ML2: $2 \mu \mathrm{M}$ ) for $72 \mathrm{~h}$ and apoptotic cell death was determined by Annexin V-FITC/PI staining and flow cytometry. $(\mathbf{b}, \mathbf{f}, \mathbf{h})$ Mean and SD of three independent experiments carried out in triplicate are shown. $* p<0.05$, $* * p<0.01, * * * p<0.001$.

(Fig. 3f) or XIAP (Fig. 3h) significantly increased SGI-110induced cell death. These results indicate that targeting of both, cIAP1 and XIAP, contributes to enhance SGI-110mediated cell death in AML cells.

\section{Sequential SGI-110/ASTX660 treatment upregulates extrinsic and intrinsic apoptosis signaling genes}

To gain further insights into the molecular mechanisms of the synergistic activity of SGI-110 and ASTX660 we performed transcriptome analysis prior to the onset of cell death. A principal component analysis was used to obtain an overview of treatment-induced changes in transcription (Fig. 4a). The first principal component (PC1) clearly separated the control and ASTX660-treated cells from those treated with SGI-110 alone or in combination with ASTX660. Analysis of DEGs revealed 2004 and 2571 DEGs upon SGI-110 alone or sequential SGI-110/ ASTX660 treatment, respectively, whereas only 158 DEGs were detectable following ASTX660 treatment compared with control (Fig. 4b). These results show that SGI-110 was primarily responsible for the global transcriptional changes following sequential SGI-110/ASTX660 treatment. We further performed GO enrichment analysis to investigate the biological functions of the identified DEGs (Fig. 4c and Supplementary Fig. S7). Interestingly, in both SGI-110 alone and sequential SGI-110/ASTX660 treatment, upregulated DEGs were significantly enriched in the apoptotic signaling pathway and IкB kinase/Nuclear factor-kappa B

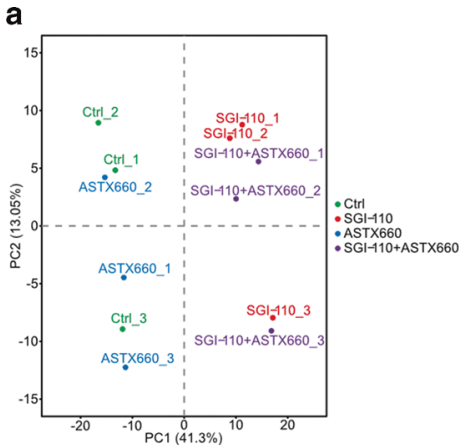

b

\begin{tabular}{|c|c|c|c|c|c|c|c|}
\hline & & \begin{tabular}{|c|c} 
AsTX660 \\
vs \\
ctrt \\
(robust)
\end{tabular} & $\begin{array}{c}\text { sol-110 } \\
\text { ss } \\
\text { ctrl }\end{array}$ & \begin{tabular}{|c|} 
SG|-110 \\
vs \\
AsTX660
\end{tabular} & \begin{tabular}{|c|} 
SGl-110+ASTX660 \\
vs \\
ctrl
\end{tabular} & $\begin{array}{c}\text { SGI-110+ASTX660 } \\
\text { vs } \\
\text { ASTX660 }\end{array}$ & $\begin{array}{c}\text { SGI-1-10+ASTX660 } \\
\text { st-110 } \\
\text { sct-110 } \\
\text { (robust) }\end{array}$ \\
\hline \multirow{3}{*}{ ADEGS } & Up & 53 & 1177 & 1113 & 1513 & 1140 & 6 \\
\hline & Down & 105 & 827 & 1299 & 1058 & 1092 & 33 \\
\hline & Total & 158 & 2004 & 2412 & 2571 & 2232 & 39 \\
\hline
\end{tabular}

C
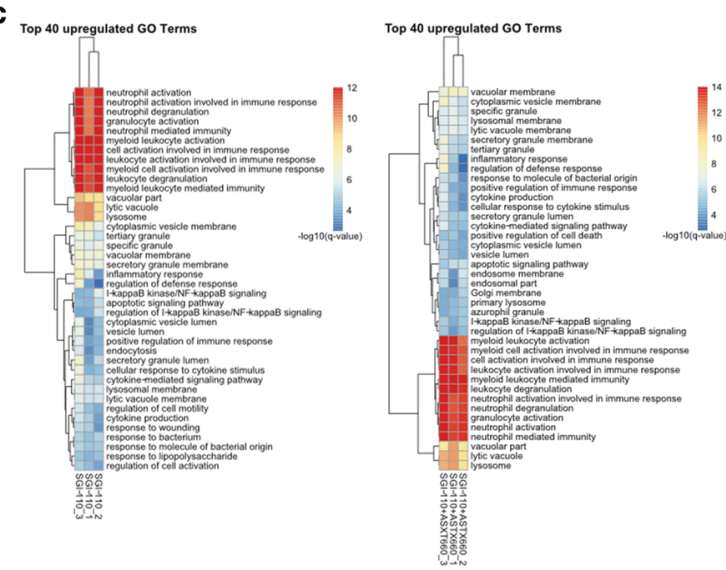

d

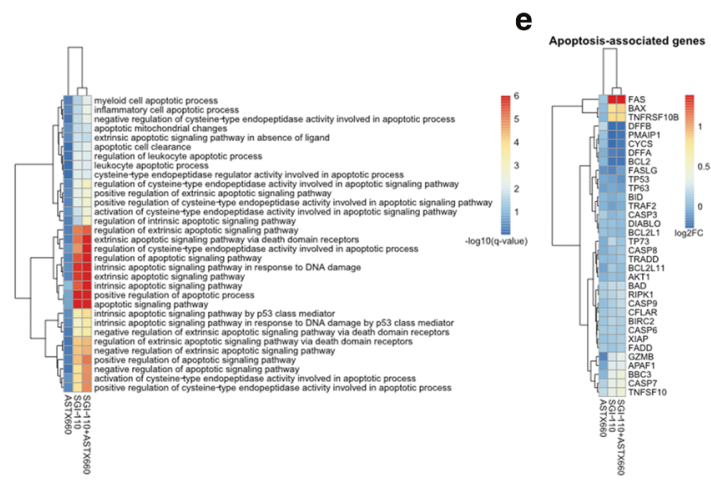

f

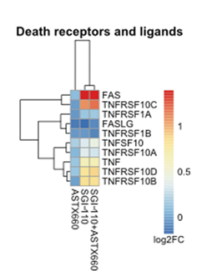

signaling. Focusing our analysis on significantly upregulated apoptotic GO terms, we found several GO terms associated with apoptosis, including the extrinsic apoptotic 
Fig. 4 Sequential SGI-110/ASTX660 treatment upregulates extrinsic and intrinsic apoptosis signaling genes. ML-2 cells were treated with solvent alone or with $2 \mu \mathrm{M}$ SGI- 110 for $24 \mathrm{~h}$ followed by 5 $\mu \mathrm{M}$ ASTX660 for $9 \mathrm{~h}$. RNA expression levels of three independent experiments were analyzed by microarray. a Principal component analysis (PCA) of ML-2 cells after drug treatment with SGI-110 and/or ASTX660 or solvent control (Ctrl). b Number of total, up-, and downregulated DEGs from multiple comparisons among the four treatment groups (adjusted $p$ value $<0.05$ ). For two comparisons, labeled with robust, the robust method for linear model fitting was used. The rest of the comparisons was fitted with the least-squares method. $\mathbf{c}$ The Top 40 enriched GO terms (biological process, cellular component, and molecular function, combined to one set for the analysis) of upregulated genes upon SGI-110 alone (left panel) or sequential SGI110/ASTX660 treatment (right panel) compared with control treatment. d Heatmap showing significantly upregulated apoptotic GO terms in at least one condition after SGI-110 and/or ASTX660 treatment, normalized to solvent-treated control cells. Pathways were considered significant with an adjusted $p$ value $<0.05$. e Heatmap showing apoptosisrelated genes regulated in SGI-110 and/or ASTX660-treated ML-2 cells, normalized to solvent-treated cells. Genes were selected based on the "Apoptosis" pathway of ConsensusPathDB and the "Apoptosis" pathway of Reactome. Only the genes annotated in both pathways were considered. f Heatmap displaying $\log 2$ fold expression changes of death receptors and their ligands upon SGI-110 and/or ASTX660 treatment compared with solvent-treated control cells. (c-f) Columns and rows were hierarchically clustered by their Euclidian distance using complete linkage. The colors correspond to the $-\log 10$ transformed adjusted $p$ values from the gene-set enrichment analysis (c, d) or $\log 2$ fold changes (FC) (e, f) obtained by comparing SGI-110 and/or ASTX660-treated versus solvent-treated control cells.

signaling pathway via death receptors, the intrinsic apoptotic signaling pathway and regulation of cysteine-type endopeptidase activity involved in apoptotic processes (Fig. 4d). Thus, we concentrated on apoptosis-related genes which were selected based on the "Apoptosis" pathway of ConsensusPathDB and Reactome (Fig. 4e). Of note, SGI110 alone or in combination with ASTX660 significantly increased gene expression of $F A S, B A X$, and TNFRSF $10 B$ (DR5). Since this analysis indicated an involvement of death receptor-mediated apoptosis, we analyzed in more detail the transcriptional changes in death receptors and their ligands (Fig. 4f). Interestingly, FAS, several TRAIL receptors (i.e., TNFRSF10B (DR5), TNFRSF10C (DcR1), and TNFRSF10D (DcR2)) as well as TNF (TNF $\alpha)$ were significantly upregulated (adjusted $p$ value $<0.05$ and $\log 2$ fold change $>0.5$ ) upon SGI-110 alone or sequential SGI110/ASTX660 treatment. With regard to the additionally observed growth-inhibitory effects of SGI-110 alone or in combination with ASTX660, we extended our GSEA on proliferation-related GO terms. Thereby, we identified the cell cycle regulator CDKN1A (p21) as the strongest upregulated gene in the most significantly enriched proliferation GO term "negative regulation of proliferation" (Supplementary Fig. S8A-B). The upregulation of CDKN1A by SGI-110 alone or when combined with ASTX660 was confirmed by qRT-PCR (Supplementary Fig. S8C). Taken together, our transcriptome analysis showed that SGI-110 alone or in combination with ASTX660 upregulated signaling pathways in extrinsic and intrinsic apoptosis and regulated genes involved in proliferative signaling.

\section{Sequential SGI-110/ASTX660 treatment initiates death receptor-dependent apoptosis}

As our transcriptome analysis identified death receptors and their ligands as top hits following SGI-110 alone or sequential SGI-110/ASTX660 treatment, we next determined the functional relevance of the death receptor pathway for SGI-110/ASTX660-induced apoptosis. Since DcR1 and DcR2 are unable to transmit death signals [41], we focused our experiments on TNFR1, DR4, DR5, FAS, and their respective ligands. Validation experiments using qRT-PCR confirmed differential expression of selected death receptors and ligands in both AML cell lines (Supplementary Fig. S9). To investigate whether these drug-induced changes in gene expression resulted in altered protein expression of death receptors we analyzed cell surface expression by flow cytometry. Indeed, SGI110 alone or sequential treatment with SGI-110 and ASTX660 significantly upregulated the surface expression of DR5 and FAS, while TNFR1 expression remained nearly unchanged (Fig. 5a, b). By comparison, DR4 was hardly detectable on the surface of MOLM-13 and ML-2 cells.

To test the functional relevance of TNFR1, FAS, and DR5 for SGI-110/ASTX660-induced apoptosis we genetically silenced these receptors by siRNA and confirmed knockdown efficiency by flow cytometry (Fig. 5c-e; Supplementary Fig. S10A-C). Importantly, knockdown of either TNFR1 or FAS significantly rescued SGI-110/ ASTX660-mediated apoptosis in both AML cell lines. By comparison, DR5 knockdown significantly protected only ML-2 cells from SGI-110/ASTX660-induced apoptosis (Fig. 5f-h; Supplementary Fig. S10D-F). This set of experiments indicates that SGI-110/ASTX660-induced cell death depended, at least partly, on death receptors such as TNFR1 and FAS.

Next, we investigated whether the observed upregulation of TNF $\alpha$ (Fig. 4f) was required for SGI-110/ASTX660mediated apoptosis as described previously for similar settings [21, 42-44]. To this end, we used the TNF $\alpha$-blocking antibody Enbrel. While Enbrel slightly reduced SGI-110/ ASTX660-induced apoptosis in ML-2 cells, it had no effect in MOLM-13 cells (Supplementary Fig. S11A). Control experiments confirmed that Enbrel significantly decreased TNF $\alpha / A S T X 660$-mediated apoptosis. In addition, TRAILor FASLG-blocking antibodies failed to rescue SGI-110/ 

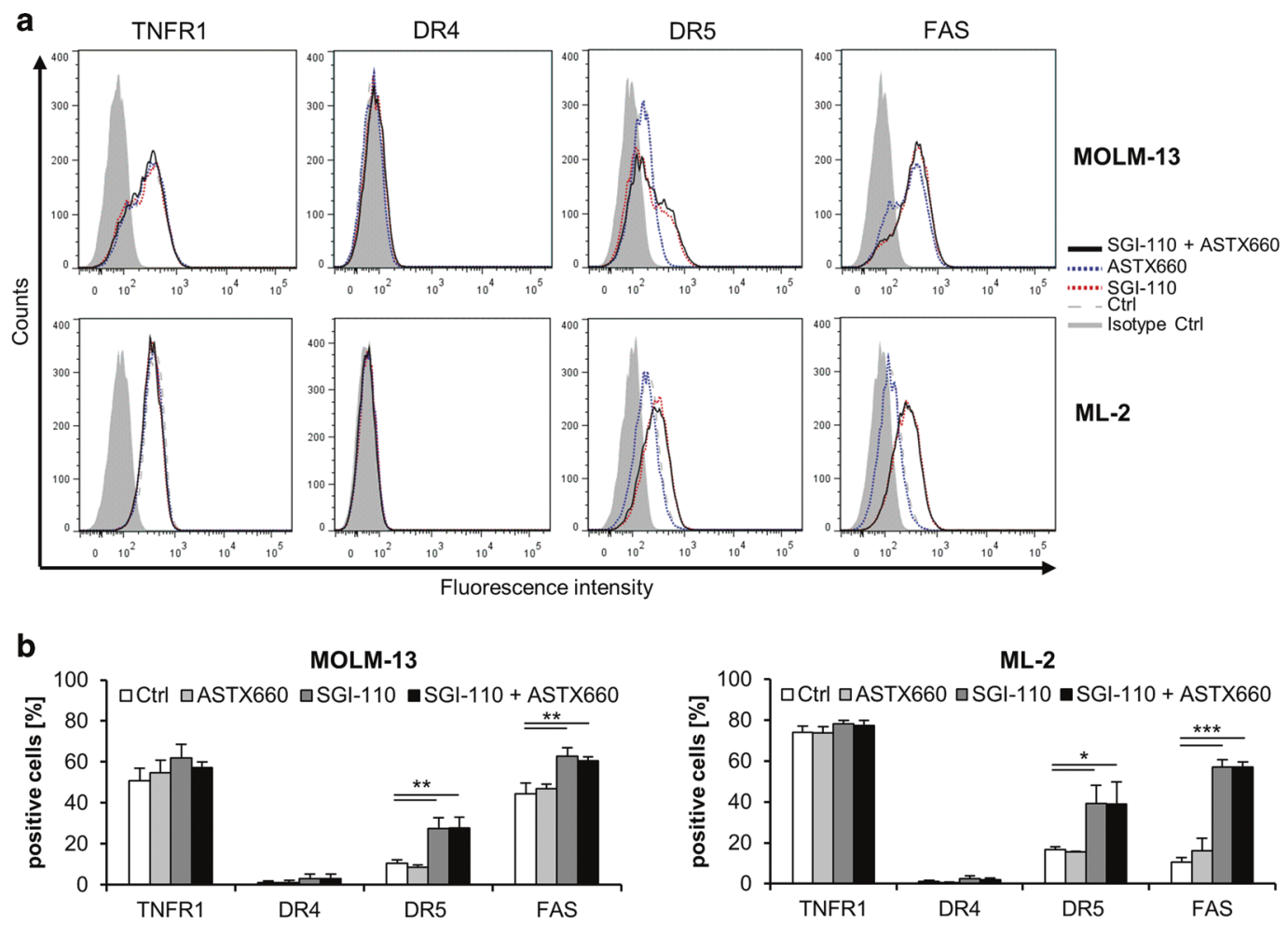

C

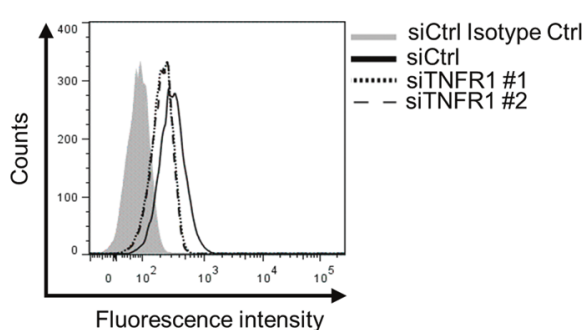

f

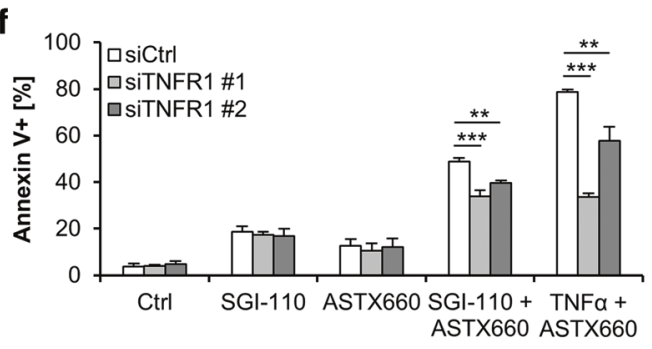

d
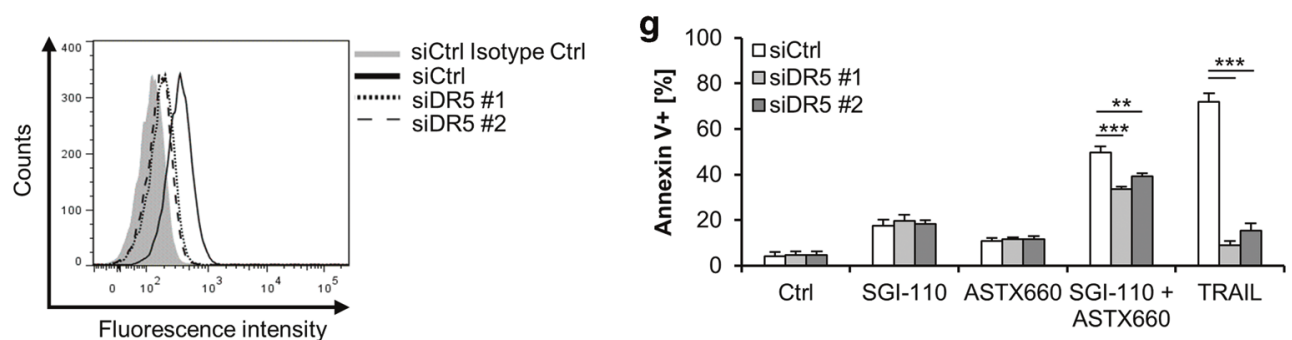

e

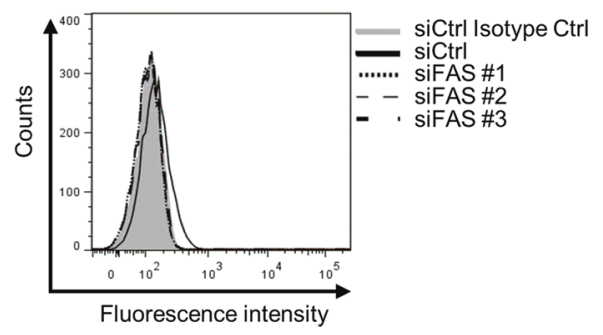

h

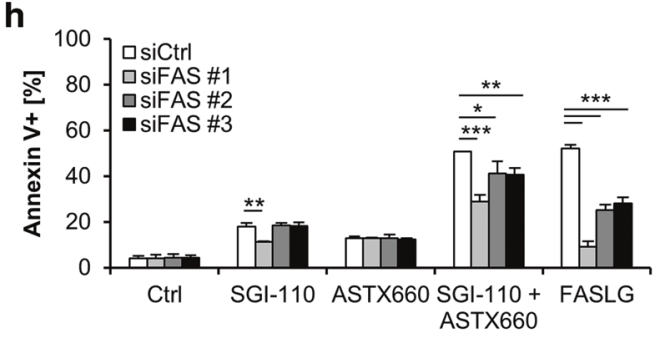

ASTX660-induced apoptosis, whereas they potently suppressed TRAIL or FASLG-mediated apoptosis used as positive controls (Supplementary Fig. S11B-C). This

indicates that SGI-110/ASTX660 induced cell death in AML cells in a TRAIL- and FASLG-independent fashion, while the effect of TNF $\alpha$ seems to be cell line-dependent. 
Fig. 5 Sequential SGI-110/ASTX660 treatment initiates death receptor-dependent apoptosis. a-b Following $24 \mathrm{~h}$ of pretreatment with SGI-110 (MOLM-13: 50 nM, ML-2: $2 \mu \mathrm{M}$ ), AML cells were treated with ASTX660 (MOLM-13: $40 \mu \mathrm{M}$, ML-2: $5 \mu \mathrm{M}$ ) for $15 \mathrm{~h}$. Death receptor expression on cell surface was determined by flow cytometric analysis after staining with PE-conjugated antibodies specific to each death receptor (open histograms) or with isotype-matched IgG controls (shaded gray histogram). Representative overlay histograms (a) and quantification of cell surface expression of death receptors (b) from three independent experiments performed in triplicate are shown. (c-h) ML-2 cells were transfected with non-targeting control siRNA (siCtrl) or siRNA against TNFR1, DR5, or FAS. (c-e) Cell surface expression of death receptors (open histograms) was analyzed by flow cytometry. Gray shaded histograms represent respective isotype controls. Representative overlay histograms are shown. (f-h) Following $24 \mathrm{~h}$ of pretreatment with $2 \mu \mathrm{M}$ SGI-110, ML2 cells were treated with $5 \mu \mathrm{M}$ ASTX660 for $36 \mathrm{~h}$. Treatments with 1 $\mathrm{ng} / \mathrm{ml}$ TNF $\alpha$ and $5 \mu \mathrm{M}$ ASTX660 (f), $10 \mathrm{ng} / \mathrm{ml}$ TRAIL (g) or $750 \mathrm{ng} /$ $\mathrm{ml}$ hexameric FAS ligand (FASLG) (h) for $36 \mathrm{~h}$ were used as positive controls to demonstrate the efficacy of gene silencing. Cell death was determined by Annexin V-FITC staining and flow cytometry. In (b, fh), mean and SD of three independent experiments performed in triplicate are shown. ${ }^{*} p<0.05,{ }^{*} p<0.01,{ }^{* * *} p<0.001$.

\section{Sequential SGI-110/ASTX660 treatment engages intrinsic apoptosis as well as a crosstalk between extrinsic and intrinsic apoptotic pathways}

As our transcriptome analysis highlighted the engagement of the intrinsic apoptosis pathway beyond the extrinsic pathway, we examined whether a crosstalk between these pathways may exist during SGI-110/ASTX660-induced apoptosis. One of the best-characterized connections between the two pathways is BID, which translocates to mitochondria after caspase-8-mediated cleavage [45]. Indeed, sequential SGI110/ASTX660 treatment-induced cleavage of caspase-8 and BID (Fig. 6a), suggesting a crosstalk between extrinsic and intrinsic apoptosis pathways.

tBID has been reported to facilitate apoptosis by engaging lysosomal permeabilization [46] in addition to promoting activation of BAX/BAK and MOMP [47]. Since our microarray analysis indicated a potential role of lysosomes upon SGI-110/ASTX660 treatment (Fig. 4c, e), we examined the effect of lysosomal protease inhibitors on SGI-110/ASTX660-induced apoptosis. However, the addition of E64D/Pepstatin A failed to rescue AML cells from SGI-110/ASTX660-induced apoptosis, while control experiments confirmed the functionality of these inhibitors (Supplementary Fig. S12).

As our microarray analysis revealed increased BAX levels by sequential SGI-110/ASTX660 treatment, we then investigated the role of BAX in SGI-110/ASTX660induced apoptosis. SGI-110/ASTX660-stimulated upregulation of BAX was confirmed by qRT-PCR (Fig. 6b) and Western blotting (Fig. 6c). To investigate whether BAX is activated upon sequential SGI-110/ASTX660 treatment we performed immunoprecipitation experiments with a conformation-specific antibody for activated BAX. Intriguingly, SGI-110 and ASTX660 acted in concert to activate BAX compared with either drug alone (Fig. 6d). To determine the functional relevance of BAX for SGI-110/ ASTX660-induced apoptosis, we performed BAX knockdown using siRNA (Fig. 6e). Notably, silencing of BAX significantly reduced SGI-110/ASTX660-mediated apoptosis (Fig. 6f). Furthermore, concomitant knockdown of BAX and BAK was significantly more effective to inhibit SGI110/ASTX660-mediated apoptosis in ML-2 cells compared with BAX knockdown alone (Fig. 6f). ABT-199 treatment, reported to kill AML cells in a BAX/BAK-dependent fashion [48], was used as a positive control (Fig. 6f).

As activated BAX can cause MOMP leading to cytochrome c release into the cytosol [49], we next assessed MMP. SGI-110 and ASTX660 cooperated to increase loss of MMP in a time-dependent manner (Fig. 6g). Together, these data suggest that sequential SGI-110/ASTX660 treatment engages intrinsic apoptosis as well as a crosstalk between extrinsic and intrinsic apoptotic pathways.

\section{SGI-110 and ASTX660 cooperate to induce biochemical and morphological hallmarks of apoptosis}

To examine effector mechanisms of SGI-110/ASTX660induced cell death we determined activation of the executioner caspases- 3 and -7 by fluorescence microscopy. Interestingly, sequential treatment with SGI-110 and ASTX660 significantly increased activation of caspases-3/7 compared with either treatment alone in a time-dependent manner (Fig. 7a). Furthermore, SGI-110 acted together with ASTX660 to significantly increase DNA fragmentation (Fig. 7b). Consistently, the analysis of nuclear morphology revealed that AML cells underwent morphological changes typical of apoptosis such as chromatin condensation and nuclear fragmentation (Fig. 7c). Quantification revealed a significant increase in nuclei fragmentation upon combination treatment compared with either single treatment or to untreated cells (Fig. 7d). Together, this set of experiments demonstrates that SGI-110 and ASTX660 cooperated to trigger typical apoptotic events such as caspase activation and nuclear fragmentation.

However, addition of the pan-caspase inhibitor zVAD. fmk alone failed to protect from SGI-110/ASTX660induced apoptosis and even increased cell death, in line with reports showing that caspase inhibition can cause a switch from apoptotic to necroptotic cell death in AML cells $[23,42]$. In line with this notion, simultaneous treatment with zVAD.fmk and the necroptosis inhibitors Necrostatin1s (Nec-1s), Necrosulfonamide (NSA), GSK'872, or Dabrafenib significantly reduced SGI-110/ASTX660-induced 
a

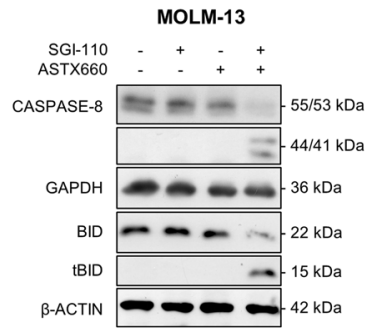

b

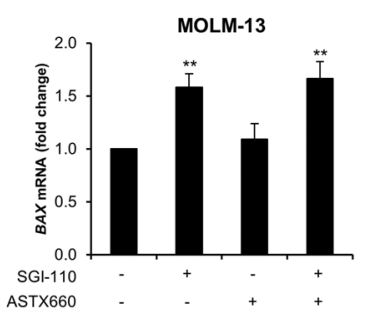

C

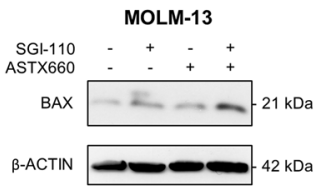

d

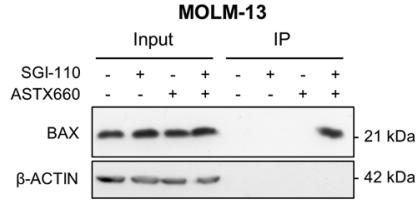

e

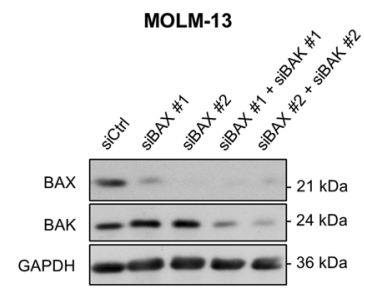

f

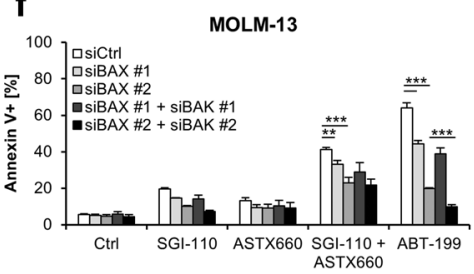

g

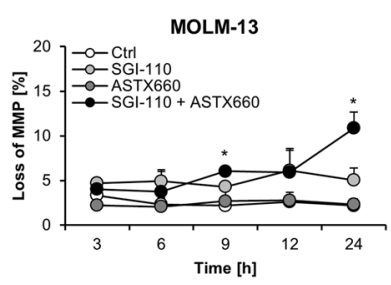

cell death compared with SGI-110/ASTX660-treated cells in the presence of zVAD.fmk alone (Supplementary Fig. S13). This indicates that SGI-110/ASTX660 primarily
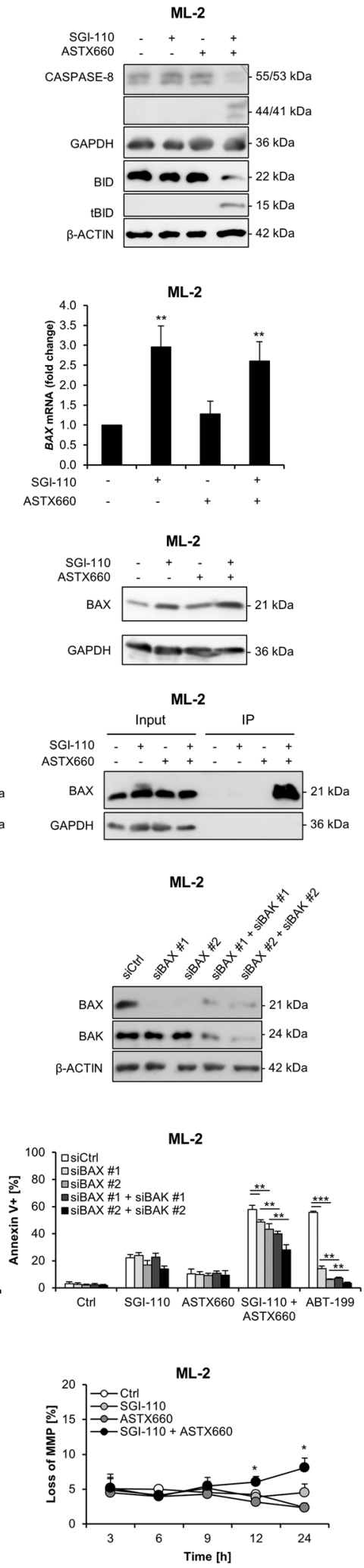

induced caspase-dependent apoptosis in apoptosisproficient AML cells, while it engaged necroptosis upon caspase inhibition. 
Fig. 6 Sequential SGI-110/ASTX660 treatment engages intrinsic apoptosis as well as a crosstalk between extrinsic and intrinsic apoptotic pathways. a AML cells were pretreated with SGI-110 (MOLM-13: $50 \mathrm{nM}$, ML-2: $2 \mu \mathrm{M}$ ) for $24 \mathrm{~h}$ followed by ASTX660 (MOLM-13: $40 \mu \mathrm{M}$, ML-2: $5 \mu \mathrm{M}$ ) for $15 \mathrm{~h}$. a Cleavage of caspase- 8 and BID was assessed by Western blotting, GAPDH, and $\beta$-ACTIN served as loading controls. b, c AML cells were pretreated with SGI110 (MOLM-13: $50 \mathrm{nM}$, ML-2: $2 \mu \mathrm{M}$ ) for $24 \mathrm{~h}$ followed by ASTX660 (MOLM-13: $40 \mu \mathrm{M}$, ML-2: $5 \mu \mathrm{M}$ ) for $9 \mathrm{~h}$ (b) or $15 \mathrm{~h}$ (c). b $B A X$ mRNA expression was analyzed by qRT-PCR and fold changes relative to untreated control are shown with mean and SD of three independent experiments performed in triplicate. Significances are calculated versus control cells. c BAX expression was determined by Western blotting, GAPDH, and $\beta$-ACTIN served as loading controls. d After $24 \mathrm{~h}$ of pretreatment with SGI-110 (MOLM-13: $50 \mathrm{nM}$, ML-2: 2 $\mu \mathrm{M}$ ), AML cells were treated with ASTX660 (MOLM-13: $40 \mu \mathrm{M}$, ML-2: $5 \mu \mathrm{M}$ ) for $15 \mathrm{~h}$ (MOLM-13) or $24 \mathrm{~h}$ (ML-2). Activation of BAX was assessed by immunoprecipitation using active conformationspecific antibodies and protein expression of BAX was detected by Western blotting. GAPDH and $\beta$-ACTIN served as loading controls. e, f AML cells were transiently transfected with siRNA against nontargeting siRNA (siCtrl) or BAX, BAK, or both. e Expression of BAX and BAK were assessed by Western blotting, with GAPDH serving as loading control. f Transfected cells were pretreated with SGI-110 (MOLM-13: $50 \mathrm{nM}$, ML-2: $2 \mu \mathrm{M}$ ) for $24 \mathrm{~h}$, followed by addition of ASTX660 (MOLM-13: $40 \mu \mathrm{M}$, ML-2: $5 \mu \mathrm{M}$ ) for $24 \mathrm{~h}$ (MOLM-13) or $36 \mathrm{~h}$ (ML-2). Treatment with $1 \mu \mathrm{M}$ ABT-199 for $24 \mathrm{~h}$ (MOLM-13) or $36 \mathrm{~h}$ (ML-2) was used as positive control to demonstrate the efficacy of gene silencing. Apoptotic cell death was measured by Annexin VFITC/PI staining and flow cytometry. $\mathrm{g}$ Following $24 \mathrm{~h}$ of pretreatment with SGI-110 (MOLM-13: 50 M, ML-2: $2 \mu \mathrm{M}$ ), AML cells were treated with ASTX660 (MOLM-13: $40 \mu \mathrm{M}$, ML-2: $5 \mu \mathrm{M}$ ) for indicated time points. Loss of MMP was analyzed by flow cytometry using the fluorescent dye TMRM. Significances after sequential SGI-110/ ASTX660 treatment are calculated versus single-treated cells. In b, f, $\mathbf{g}$, mean and SD of three independent experiments carried out in triplicate are shown. ${ }^{*} p<0.05,{ }^{* *} p<0.01, * * * p<0.001$.

\section{Discussion}

Given the limited treatment options for elderly patients, the development of more effective and well-tolerated therapies remains a major unmet medical need [50]. Approval of decitabine for the first-line treatment of elderly AML patients highlights the general potential of HMAs in this setting [51]. However, as HMAs demonstrated only limited efficacy as monotherapy, combination strategies with either chemotherapy, immunotherapy, or targeted therapies such as the BCL-2 inhibitor ABT-199 are currently in different stages of clinical testing [13]. As evasion of apoptosis, e.g., by overexpression of IAPs, contributes to treatment resistance and poor outcome in AML [52], in the present study we investigated the combination of SGI-110 and the IAP antagonist ASTX660. Here, we discover that the combination of SGI-110 and ASTX660 synergistically induced cell death in a range of AML cell lines as well as in patientderived AML blasts. By comparison, sequential treatment with SGI-110 and ASTX660 exhibited limited toxicity to normal CD34+ HPCs, pointing to a favorable therapeutic window. Concentrations of SGI-110 $(0.1-1 \mu \mathrm{M})$ required for synergism with ASTX660 in our study might be achievable in AML patients, as clinically achievable plasma levels have been reported to range from 20 to $400 \mathrm{nM}$ for SGI-110 and from 0.3 to $1.6 \mu \mathrm{M}$ for parental compound decitabine [37, 53].

While the DNMT3A mutant AML cell line OCI-AML3 proved to be resistant to sequential SGI-110/ASTX660 treatment, SGI-110 together with ASTX660 synergistically induced cell death in the tested primary AML sample harboring DNMT3A mutation. In line with this finding, Metzeler et al. reported that DNMT3A-mutated patients benefit from HMA treatment [54]. A possible explanation might be that $D N M T 3 A^{\mathrm{R} 882}$-associated hypomethylation patterns are preserved in primary AML samples, but not in the $D N M T 3 A^{\mathrm{R} 882 \mathrm{C}}$-mutated AML cell line OCI-AML3 [55]. Further studies are required to unravel the mechanisms for SGI-110/ASTX660 resistance in OCI-AML3 cells.

Importantly, our study provides new insights into the molecular mechanisms underlying the synergistic induction of cell death by SGI-110 and ASTX660. We identify activation of both extrinsic and intrinsic apoptotic pathways as key events during SGI-110/ASTX660-induced cell death in AML cells. This conclusion is supported by several lines of evidence.

First, unbiased transcriptome analysis revealed that SGI110 alone and sequential SGI-110/ASTX660 treatment upregulated the death receptors $D R 5$ and $F A S$, which was accompanied by increased protein expression on the cell surface. FAS upregulation might be a direct effect of HMAinduced FAS promoter demethylation [56]. By comparison, DR4 was hardly detectable on the cell surface of both AML cell lines, which is in line with previous studies demonstrating the absence or low expression of DR4 for different cancer entities [57, 58]. Second, TNFR1, DR5, and FAS were all required for cell death induction, since individual silencing of these death receptors significantly reduced SGI110/ASTX660-induced apoptosis. The fact that SGI-110/ ASTX660-mediated cell death did not depend on a single death receptor underlines the importance of the death receptor pathway as a whole rather than single gene activation. Third, experiments with blocking antibodies revealed a contribution of TNF $\alpha$-driven autocrine/paracrine signaling to SGI-110/ASTX660-mediated apoptosis in a cell line-dependent manner, which is consistent with our previous studies showing that Enbrel rescued leukemia cells from HMA/IAP antagonist-induced cell death [42, 43]. However, SGI-110/ASTX660-induced cell death occurred independently of TRAIL and FASLG, suggesting an activation of DR5 and FAS in a ligand-independent fashion. This is in line with previous evidence suggesting that anticancer drug-mediated apoptosis involved ligandindependent death receptor clustering and activation of caspases in leukemia as well as solid cancer cells [59-61]. 
a

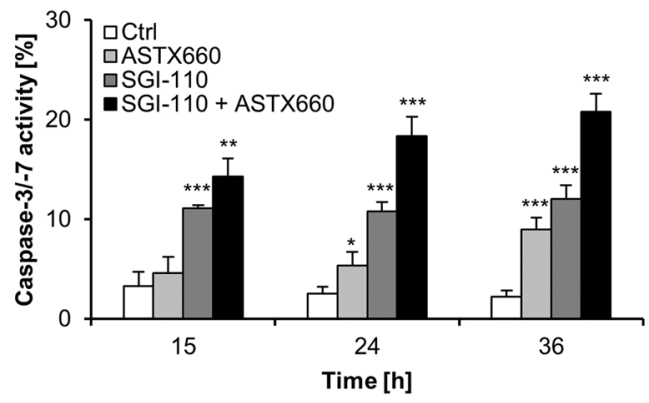

b

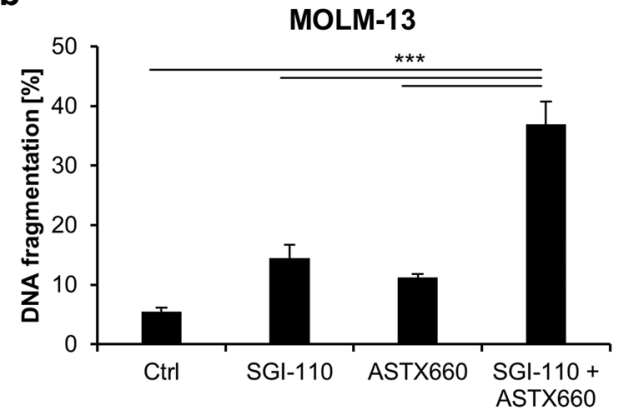

ML-2

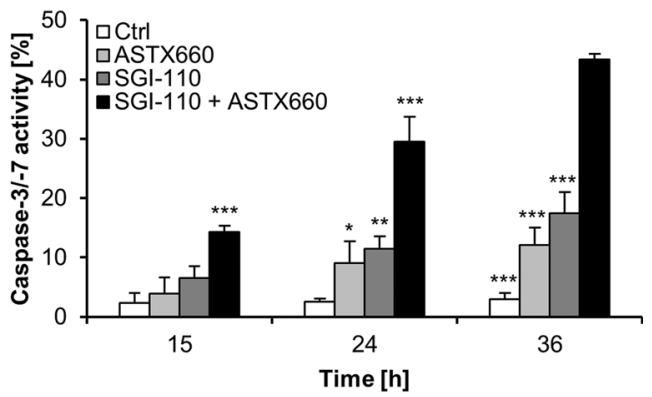

ML-2

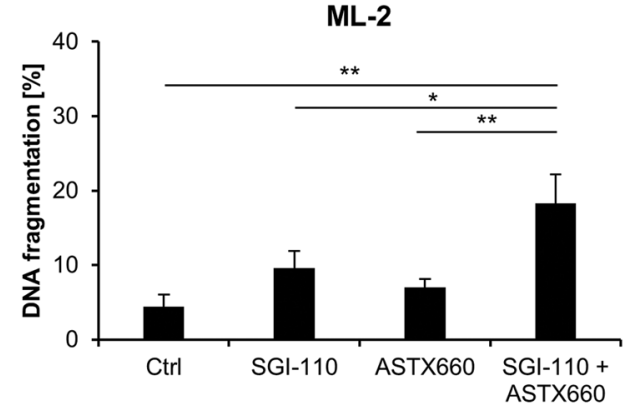

C

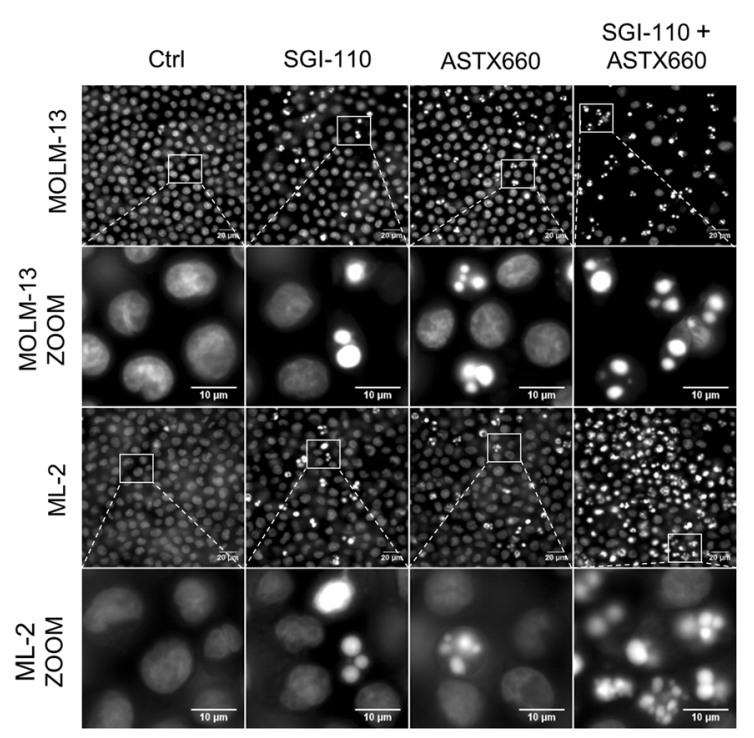

d

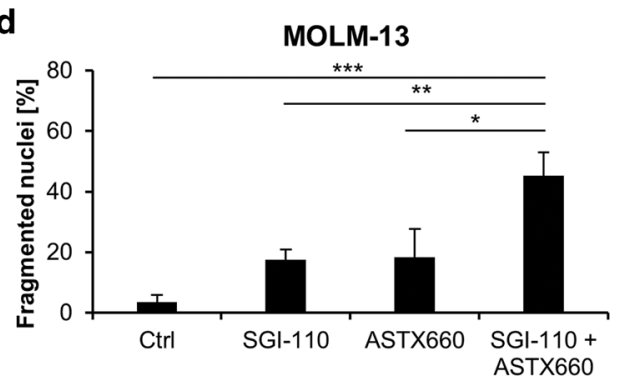

Fourth, SGI-110/ASTX660-induced death signals are transmitted by caspase- 8 and tBID to mitochondria, leading to activation of BAX and finally mitochondrial apoptosis. Fifth, SGI-110 increases BAX expression, in line with a

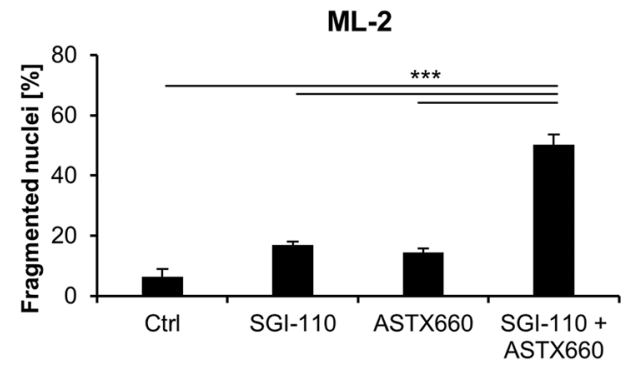

previous study using decitabine [62], thus shifting the balance of pro- and antiapoptotic factors towards apoptosis. The crucial role of BAX for SGI-110/ASTX660-induced apoptosis is emphasized by genetic silencing of BAX, 
Fig. 7 SGI-110 and ASTX660 cooperate to induce biochemical and morphological hallmarks of apoptosis. a After $24 \mathrm{~h}$ of pretreatment with SGI-110 (MOLM-13: $50 \mathrm{nM}$, ML-2: $2 \mu \mathrm{M}$ ), AML cells were additionally treated with ASTX660 (MOLM-13: $40 \mu \mathrm{M}$, ML-2: $5 \mu \mathrm{M})$ for indicated time points. Caspase-3/-7 activity was determined by Cell Event Caspase-3/-7 Green Detection Reagent and ImageXpress Micro XLS system. Significances are calculated versus control cells. b-d Following 24 h of SGI-110 pretreatment (MOLM-13: 50 nM, ML-2: $2 \mu \mathrm{M}$ ), AML cells were treated with ASTX660 (MOLM13: $40 \mu \mathrm{M}$, ML-2: $5 \mu \mathrm{M}$ ) for $48 \mathrm{~h}$. b DNA fragmentation was analyzed by flow cytometry after PI staining of nuclei. c Nuclear fragmentation was imaged using Hoechst-33342 staining and ImageXpress Micro XLS system. Representative images per sample are shown. d Quantification of fragmented nuclei upon indicated treatment. Mean and SD of three independent experiments performed in triplicate are shown. $* p<0.05, * * p<0.01, * * * p<0.001$

which significantly protected AML cells from SGI-110/ ASTX660-mediated apoptosis. Taken together, our in-depth molecular studies highlight the importance of both extrinsic and intrinsic apoptotic pathways in mediating SGI-110/ ASTX660-induced apoptosis.

Since both SGI-110 and ASTX660 as single agents are currently under evaluation in early clinical trials, our findings have important implications for the development of new combination strategies for AML. SGI-110 may offer several advantages compared with first-generation HMAs, as subcutaneous administration of SGI-110 exhibited increased efficacy and reduced toxicity compared with decitabine due to reduced peak plasma levels and prolonged half-life [8]. In addition, response rates to SGI-110 were reported in AML patients after azacytidine failure [63]. Previously, only first-generation HMAs such as azacytidine and decitabine have been shown to synergistically induce cell death together with IAP antagonist in AML [42], acute lymphoblastic leukemia [43] as well as in many NCI-60 cell lines from different cancer types [64], indicating a potential broad application of this combination strategy in cancer therapy. The success of current antileukemic therapies is often limited by evasion of apoptosis [52] highlighting the potential of this combination strategy of SGI-110 and ASTX660 for the treatment of AML.

Acknowledgements We thank S. Jordan (UCT Biobank, Frankfurt) for providing primary AML samples, H. Bönig (Institute for Transfusion Medicine and Immunohematology, University Hospital Frankfurt) for providing CD34+ HPCs, Astex Pharmaceuticals (Cambridge, UK) for providing SGI-110 and ASTX660, C. Hugenberg for expert secretarial assistance and Dr M. Bewerunge-Hudler and her team (Genomics and Proteomics Core Facility, German Cancer Research Center/DKFZ, Heidelberg, Germany) for their microarray service. We thank V. Ehrenfeld for assistance with image preparation. This work was supported by grants from the BMBF (to SF) and German Cancer Aid (to SF), and by Astex Pharmaceuticals, Cambridge, UK (to SF). MB is supported by grants from the DFG (CRC850), BMBF (CoNfirm; FKZ 01ZX1708F), and MIRACUM within the Medical Informatics Funding Scheme (FKZ 01ZZ1606A$\mathrm{H})$. PM is funded by MIRACUM.

\section{Compliance with ethical standards}

Conflict of interest This work was supported in part by Astex Pharmaceuticals, Cambridge, UK (to SF). GAW is employee of Astex Pharmaceuticals. JD, TH, PM, MB, and MV declare no conflict of interest.

Publisher's note Springer Nature remains neutral with regard to jurisdictional claims in published maps and institutional affiliations.

\section{References}

1. O'Donnell MR, Tallman MS, Abboud CN, Altman JK, Appelbaum FR, Arber DA, et al. Acute myeloid leukemia, version 3.2017, NCCN clinical practice guidelines in oncology. J Natl Compr Canc Netw. 2017;15:926-57.

2. Dohner H, Weisdorf DJ, Bloomfield CD. Acute myeloid leukemia. N. Engl J Med. 2015;373:1136-52.

3. Chaudry SF, Chevassut TJ. Epigenetic guardian: a review of the DNA methyltransferase DNMT3A in acute myeloid leukaemia and clonal haematopoiesis. Biomed Res Int 2017;2017:5473197.

4. Marcucci G, Metzeler KH, Schwind S, Becker H, Maharry K, Mrozek K, et al. Age-related prognostic impact of different types of DNMT3A mutations in adults with primary cytogenetically normal acute myeloid leukemia. J Clin Oncol. 2012;30:742-50.

5. Nieto M, Demolis P, Béhanzin E, Moreau A, Hudson I, Flores B, et al. The European Medicines Agency review of decitabine (Dacogen) for the treatment of adult patients with acute myeloid leukemia: summary of the scientific assessment of the committee for medicinal products for human use. Oncologist. 2016;21:692-700.

6. Griffiths E, Choy G, Redkar S, Taverna P, Azab M, Karpf AR. SGI-110: DNA methyltransferase inhibitor oncolytic. Drugs future. 2013;38:535

7. Roboz GJ, Kantarjian HM, Yee KWL, Kropf PL, O'Connell CL, Griffiths EA, et al. Dose, schedule, safety, and efficacy of guadecitabine in relapsed or refractory acute myeloid leukemia. Cancer. 2018;124:325-34.

8. Kantarjian HM, Roboz GJ, Kropf PL, Yee KWL, O'Connell CL, Tibes R, et al. Guadecitabine (SGI-110) in treatment-naive patients with acute myeloid leukaemia: phase 2 results from a multicentre, randomised, phase 1/2 trial. Lancet Oncol. 2017;18: 1317-26.

9. Wolff F, Leisch M, Greil R, Risch A, Pleyer L. The double-edged sword of (re)expression of genes by hypomethylating agents: from viral mimicry to exploitation as priming agents for targeted immune checkpoint modulation. Cell Commun Signal. 2017; 15:13.

10. Fang F, Munck J, Tang J, Taverna P, Wang Y, Miller DF, et al. The novel, small-molecule DNA methylation inhibitor SGI-110 as an ovarian cancer chemosensitizer. Clin Cancer Res. 2014;20: 6504-16.

11. Kuang Y, El-Khoueiry A, Taverna P, Ljungman M, Neamati N. Guadecitabine (SGI-110) priming sensitizes hepatocellular carcinoma cells to oxaliplatin. Mol Oncol. 2015;9:1799-814.

12. Srivastava P, Paluch BE, Matsuzaki J, James SR, Collamat-Lai G, Karbach J, et al. Immunomodulatory action of SGI-110, a hypomethylating agent, in acute myeloid leukemia cells and xenografts. Leuk Res. 2014;38:1332-41.

13. Bewersdorf JP, Shallis R, Stahl M, Zeidan AM. Epigenetic therapy combinations in acute myeloid leukemia: what are the options? Ther Adv Hematol. 2019;10:2040620718816698.

14. Fulda S. Cell death in hematological tumors. Apoptosis. 2009;14: 409-23. 
15. Ashkenazi A. Targeting the extrinsic apoptosis pathway in cancer. Cytokine Growth Factor Rev. 2008;19:325-31.

16. Li H, Zhu H, Xu C-j, Yuan J. Cleavage of BID by caspase 8 mediates the mitochondrial damage in the Fas pathway of apoptosis. Cell. 1998;94:491-501.

17. Fulda S, Vucic D. Targeting IAP proteins for therapeutic intervention in cancer. Nat Rev Drug Discov. 2012;11:109-24.

18. Tamm I, Richter S, Scholz F, Schmelz K, Oltersdorf D, Karawajew L, et al. XIAP expression correlates with monocytic differentiation in adult de novo AML: impact on prognosis. Hematol J. 2004;5:489-95.

19. Wuchter C, Richter S, Oltersdorf D, Karawajew L, Ludwig WD, Tamm I. Differences in the expression pattern of apoptosis-related molecules between childhood and adult de novo acute myeloid leukemia. Haematologica. 2004;89:363-4.

20. Eckelman BP, Salvesen GS, Scott FL. Human inhibitor of apoptosis proteins: why XIAP is the black sheep of the family. EMBO Rep. 2006;7:988-94.

21. Varfolomeev E, Blankenship JW, Wayson SM, Fedorova AV, Kayagaki N, Garg P, et al. IAP antagonists induce autoubiquitination of c-IAPs, NF-kappaB activation, and TNFalphadependent apoptosis. Cell. 2007;131:669-81.

22. Ward GA, Lewis EJ, Ahn JS, Johnson CN, Lyons JF, Martins V, et al. ASTX660, a novel non-peptidomimetic antagonist of cIAP1/ 2 and XIAP, potently induces TNFalpha-dependent apoptosis in cancer cell lines and inhibits tumor growth. Mol Cancer Ther. 2018;17:1381-91.

23. Safferthal C, Rohde K, Fulda S. Therapeutic targeting of necroptosis by Smac mimetic bypasses apoptosis resistance in acute myeloid leukemia cells. Oncogene. 2017;36:1487-502.

24. Lacombe F, Durrieu F, Briais A, Dumain P, Belloc F, Bascans E, et al. Flow cytometry CD45 gating for immunophenotyping of acute myeloid leukemia. Leukemia 1997;11:1878-86.

25. Nicoletti I, Migliorati G, Pagliacci MC, Grignani F, Riccardi C. A rapid and simple method for measuring thymocyte apoptosis by propidium iodide staining and flow cytometry. J Immunol Methods. 1991;139:271-9.

26. Gentleman RC, Carey VJ, Bates DM, Bolstad B, Dettling M, Dudoit $\mathrm{S}$, et al. Bioconductor: open software development for computational biology and bioinformatics. Genome Biol 2004;5:R80.

27. Huber W, Carey VJ, Gentleman R, Anders S, Carlson M, Carvalho BS, et al. Orchestrating high-throughput genomic analysis with Bioconductor. Nat Methods. 2015;12:115-21.

28. Gautier L, Cope L, Bolstad BM, Irizarry RA. affy-analysis of Affymetrix GeneChip data at the probe level. Bioinformatics. 2004;20:307-15.

29. Smyth GK, Michaud J, Scott HS. Use of within-array replicate spots for assessing differential expression in microarray experiments. Bioinformatics. 2005;21:2067-75.

30. Luo W, Friedman MS, Shedden K, Hankenson KD, Woolf PJ. GAGE: generally applicable gene set enrichment for pathway analysis. BMC Bioinf. 2009;10:161.

31. Ashburner M, Ball CA, Blake JA, Botstein D, Butler H, Cherry JM, et al. Gene ontology: tool for the unification of biology. The Gene Ontology Consortium. Nat Genet. 2000;25:25-29.

32. Gene Ontology Consortium. Gene Ontology Consortium: going forward. Nucleic Acids Res. 2015;43:D1049-1056.

33. Fabregat A, Jupe S, Matthews L, Sidiropoulos K, Gillespie M, Garapati $\mathrm{P}$, et al. The reactome pathway knowledgebase. Nucleic Acids Res. 2018;46:D649-D655.

34. Kamburov A, Stelzl U, Lehrach H, Herwig R. The ConsensusPathDB interaction database: 2013 update. Nucleic Acids Res. 2013;41:D793-800.

35. Chou T. The median-effect principle and the combination index for quantitation of synergism and antagonism. In: Chou TC and
Rideout DC, editors. Synergism and antagonism in chemotherapy, Academic Press, San Diego, CA, 1991. 61-102.

36. Scholzen T, Gerdes J. The Ki-67 protein: from the known and the unknown. J Cell Physiol. 2000;182:311-22.

37. Issa JJ, Roboz G, Rizzieri D, Jabbour E, Stock W, O’Connell C, et al. Safety and tolerability of guadecitabine (SGI-110) in patients with myelodysplastic syndrome and acute myeloid leukaemia: a multicentre, randomised, dose-escalation phase 1 study. Lancet Oncol. 2015;16:1099-110.

38. Weinstein HJ, Griffin TW, Feeney J, Cohen HJ, Propper RD, Sallan SE. Pharmacokinetics of continuous intravenous and subcutaneous infusions of cytosine arabinoside. Blood. 1982;59:1351-3.

39. Sampath D, Cortes J, Estrov Z, Du M, Shi Z, Andreeff M, et al. Pharmacodynamics of cytarabine alone and in combination with 7-hydroxystaurosporine (UCN-01) in AML blasts in vitro and during a clinical trial. Blood. 2006;107:2517-24.

40. Yang AS, Estecio MR, Doshi K, Kondo Y, Tajara EH, Issa JP. A simple method for estimating global DNA methylation using bisulfite PCR of repetitive DNA elements. Nucleic Acids Res. 2004;32:e38.

41. Marsters SA, Pitti RA, Sheridan JP, Ashkenazi A. Control of apoptosis signaling by Apo2 ligand. Recent Prog Horm Res. 1999;54:225-34.

42. Steinhart L, Belz K, Fulda S. Smac mimetic and demethylating agents synergistically trigger cell death in acute myeloid leukemia cells and overcome apoptosis resistance by inducing necroptosis. Cell Death Dis. 2013;4:e802.

43. Gerges S, Rohde K, Fulda S. Cotreatment with Smac mimetics and demethylating agents induces both apoptotic and necroptotic cell death pathways in acute lymphoblastic leukemia cells. Cancer Lett. 2016;375:127-32.

44. Chromik J, Safferthal C, Serve H, Fulda S. Smac mimetic primes apoptosis-resistant acute myeloid leukaemia cells for cytarabineinduced cell death by triggering necroptosis. Cancer Lett. 2014;344:101-9.

45. Billen LP, Shamas-Din A, Andrews DW. Bid: a Bax-like BH3 protein. Oncogene. 2008;27:S93-104.

46. Guicciardi ME, Bronk SF, Werneburg NW, Yin X-M, Gores GJ. Bid is upstream of lysosome-mediated caspase 2 activation in tumor necrosis factor $\alpha$-induced hepatocyte apoptosis. Gastroenterology 2005;129:269-84.

47. Delbridge AR, Grabow S, Strasser A, Vaux DL. Thirty years of BCL-2: translating cell death discoveries into novel cancer therapies. Nat Rev Cancer. 2016;16:99-109.

48. Luedtke DA, Niu X, Pan Y, Zhao J, Liu S, Edwards H, et al. Inhibition of Mcl-1 enhances cell death induced by the Bcl-2selective inhibitor ABT-199 in acute myeloid leukemia cells. Signal Transduct Target Ther. 2017;2:17012.

49. Garrido C, Galluzzi L, Brunet M, Puig PE, Didelot C, Kroemer G. Mechanisms of cytochrome $\mathrm{c}$ release from mitochondria. Cell Death Differ. 2006;13:1423-33.

50. Bell JA, Galaznik A, Huelin R, Stokes M, Guo Y, Fram RJ, et al. Effectiveness and safety of therapeutic regimens for elderly patients with acute myeloid leukemia: a systematic literature review. Clin Lymphoma Myeloma Leuk. 2018;18:e303-e314.

51. He P-F, Zhou J-D, Yao D-M, Ma J-C, Wen X-M, Zhang Z-H, et al. Efficacy and safety of decitabine in treatment of elderly patients with acute myeloid leukemia: a systematic review and meta-analysis. Oncotarget. 2017;8:41498.

52. Fulda S. Exploiting inhibitor of apoptosis proteins as therapeutic targets in hematological malignancies. Leukemia. 2012;26:1155-65.

53. Hollenbach PW, Nguyen AN, Brady H, Williams M, Ning Y, Richard $\mathrm{N}$, et al. A comparison of azacitidine and decitabine activities in acute myeloid leukemia cell lines. PLoS ONE. 2010; 5:e9001. 
54. Metzeler KH, Walker A, Geyer S, Garzon R, Klisovic RB, Bloomfield CD, et al. DNMT3A mutations and response to the hypomethylating agent decitabine in acute myeloid leukemia. Leukemia. 2011;26:1106.

55. Chen D, Christopher M, Helton NM, Ferguson I, Ley TJ, Spencer DH. DNMT3A(R882)-associated hypomethylation patterns are maintained in primary AML xenografts, but not in the DNMT3A (R882C) OCI-AML3 leukemia cell line. Blood Cancer J. 2018;8:38.

56. Karlic H, Herrmann H, Varga F, Thaler R, Reitermaier R, Spitzer $\mathrm{S}$, et al. The role of epigenetics in the regulation of apoptosis in myelodysplastic syndromes and acute myeloid leukemia. Crit Rev Oncol Hematol. 2014;90:1-16.

57. Chen J-J, Shen HCJ, Rivera Rosado LA, Zhang Y, Di X, Zhang B. Mislocalization of death receptors correlates with cellular resistance to their cognate ligands in human breast cancer cells. Oncotarget. 2012;3:833-42.

58. Bousserouel S, Le Grandois J, Gosse F, Werner D, Barth SW, Marchioni E, et al. Methanolic extract of white asparagus shoots activates TRAIL apoptotic death pathway in human cancer cells and inhibits colon carcinogenesis in a preclinical model. Int $\mathbf{J}$ Oncol. 2013;43:394-404.
59. Bush JA, Cheung KJ Jr., Li G. Curcumin induces apoptosis in human melanoma cells through a Fas receptor/caspase-8 pathway independent of p53. Exp Cell Res. 2001;271:305-14.

60. Day TW, Huang S, Safa AR. c-FLIP knockdown induces ligandindependent DR5-, FADD-, caspase-8-, and caspase-9-dependent apoptosis in breast cancer cells. Biochem Pharmacol. 2008;76:1694-704.

61. Micheau O, Solary E, Hammann A, Dimanche-Boitrel MT. Fas ligand-independent, FADD-mediated activation of the Fas death pathway by anticancer drugs. J Biol Chem. 1999;274:7987-92.

62. Shin DY, Park YS, Yang K, Kim GY, Kim WJ, Han MH, et al. Decitabine, a DNA methyltransferase inhibitor, induces apoptosis in human leukemia cells through intracellular reactive oxygen species generation. Int J Oncol. 2012;41:910-8.

63. Sebert M, Renneville A, Bally C, Peterlin P, Beyne-Rauzy O, Legros L, et al. A phase II study of guadecitabine in higher-risk myelodysplastic syndrome and low blast count acute myeloid leukemia after azacitidine failure. Haematologica. 2019;104:1565-71.

64. Carter BZ, Mak PY, Mak DH, Shi Y, Qiu Y, Bogenberger JM, et al. Synergistic targeting of AML stem/progenitor cells with IAP antagonist birinapant and demethylating agents. J Natl Cancer Inst. 2014;106:djt440. 